\title{
Synthesis of 5-Fluoroaminobenzothiazoles
}

\author{
Pooja Sharma \\ Gurdial Singh*a (i) \\ Bhoomendra A. Bhongade ${ }^{a, b}$ \\ Malleshappa N. Noolvic \\ Andanappa K. Gadad \\ a Department of Chemistry, The University of The West Indies, \\ St. Augustine, Trinidad and Tobago \\ gurdial.singh@sta.uwi.edu \\ b RAK College of Pharmaceutical Sciences, RAK Medical \& \\ Health Sciences University, Ras Al Khaimah, UAE \\ c Shree Dhanvantary Pharmacy College, Surat, Gujarat, India \\ d School of Pharmacy, Faculty of Medical Sciences, The Universi- \\ ty of The West Indies, Mount Hope, Trinidad and Tobago
}

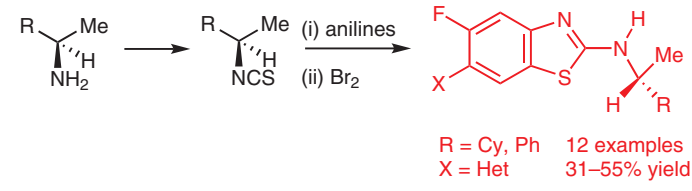

Similarly, several reports have described the anticancer $^{17}$ and anti-TB ${ }^{17 c, 18}$ activities of various $\mathrm{N}$-substituted thiourea derivatives. Of particular interest to us were the observations that 2-(N-substituted)aminobenzothiazole derivatives $1\left(\mathrm{R}=\mathrm{Cl}, \mathrm{Br}, \mathrm{Me}, \mathrm{NO}_{2}\right.$, NHAc) and thiourea derivative $\mathbf{2}$ (Figure 1) exhibited promising anti-TB ${ }^{16 c}$ and antitumor $^{17 \mathrm{~b}}$ activities, respectively.

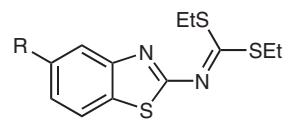

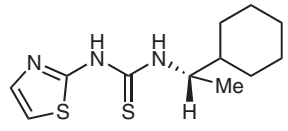

Figure 1

Key words synthetic methods, 2-aminobenzothiazoles, isothiocyanates, chiral, fluoro, heterocyclic

In 2018, the WHO reported that there were 10 million new cases of tuberculosis (TB) and it remains among the leading causes of the mortality and morbidity worldwide. ${ }^{1}$ The discovery of several potent anti-TB agents was made about 65 years and, since then, a number of agents have been discovered. ${ }^{2}$ The emergence of multi-drug resistant strains of TB bacillus to most of the currently used anti-TB drugs combined with their toxicity and side effects are of major concern in the treatment of TB. ${ }^{3}$ Thus, successful treatment of TB demands the synthesis of new, safer and more effective agents.

Benzothiazoles are an important class of biodynamic heterocyclic compounds in medicinal chemistry because of their applications in drug discovery and development. ${ }^{4}$ In particular, 2-aminobenzothiazole derivatives are reported to exhibit a wide range of biological activities such as antidiabetic, $^{5}$ antiepileptic, ${ }^{6}$ analgesic, ${ }^{7}$ antiinflammatory, ${ }^{7 c, 8}$ anthelmintic, ${ }^{9}$ antiviral, ${ }^{10}$ antifungal, ${ }^{11}$ anesthetic, ${ }^{7 c, 12}$ antiproliferative, ${ }^{13}$ antimicrobial, ${ }^{14}$ anticancer ${ }^{15}$ and antitubercular action. ${ }^{16}$ 
Molecules containing fluorine atoms are at the leading edge of many new developments in medicinal chemistry, resulting in an increased number of fluorinated organic molecules finding efficacy in the clinic. ${ }^{19}$ Selective introduction of a fluorine atom into biomolecules often results in improved potency compared to their non-fluorinated analogues, primarily due to significant improvements in their physicochemical properties. ${ }^{20}$ Over the last two decades, the development of enantiomerically pure drugs has become a major focus of most pharmaceutical companies because of their improved safety, efficacy and minimized side effects. $^{21-24}$

In considerations of these findings, and in continuation of our research in the development of novel bioactive molecules, ${ }^{25}$ we report the synthesis of optically active thiourea and N-substituted 5-fluoroaminobenzothiazoles derivatives and their preliminary in vitro evaluation as anti-TB agents.

The general synthetic strategy employed for the synthesis of the optically active isothiocyanates $\mathbf{5}$ and 6-substituted 5-fluoro-2-(N-substituted)aminobenzothiazole derivatives $\mathbf{6}$ is summarised in Scheme 1. The optically active isothiocyanates $\mathbf{5}$ were prepared by the reaction of appropriate chiral amines $\mathbf{4}$ with thiophosgene in aqueous $\mathrm{CH}_{2} \mathrm{Cl}_{2}$ in the presence of $\mathrm{NaHCO}_{3}$ at room temperature. ${ }^{26}$ Treatment of the appropriate isothiocyanates 5 with 4-substituted-3-fluoroaniline in methanol under an inert atmosphere yielded the requisite thioureas. Intramolecular oxidative cyclisation of these using bromine in chloroform afforded the corresponding 5-fluoroaminobenzothiazoles 6 in $31-55 \%$ yields.

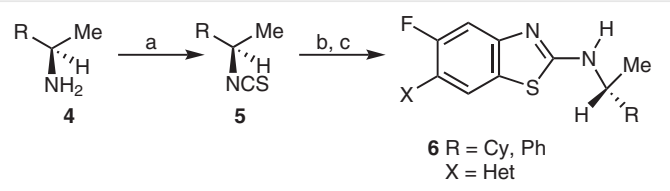

Scheme 1 Reagents and conditions: (a) $\mathrm{CSCl}_{2}, \mathrm{CH}_{2} \mathrm{Cl}_{2}, \mathrm{H}_{2} \mathrm{O}$; $\mathrm{NaHCO}_{3}$; (b) 4-substituted 3-fluoroaniline, $\mathrm{MeOH}, \mathrm{N}_{2}$, reflux; (c) $\mathrm{Br}_{2}, \mathrm{CHCl}_{3}$, r.t.

The ${ }^{1} \mathrm{H}$ NMR spectra for $\mathrm{N}$-substituted 5-fluoroaminobenzothiazoles $\mathbf{6}$ showed corresponding resonances in the range of $\delta=1.25-1.0 \mathrm{ppm}$ for the methyl group, while the chiral proton was found to resonate in the range of $\delta=4.85-$ $4.60 \mathrm{ppm}$. In their ${ }^{13} \mathrm{C}$ NMR spectra, resonances in the range of $\delta=60-49$ and 25-22 ppm were observed for chiral and methyl carbons, respectively. ${ }^{27}$

The required $\mathrm{N}$-substituted piperazine $\mathbf{1 0}$ was prepared as detailed in Scheme 2, in a two-step procedure from the commercially available 1,2-difluoro-4-nitrobenzene 7 . The resulting nitro derivative $\mathbf{9}$ was reduced to the correspond- ing aniline, which, on coupling with the chiral isothiocyanate $\mathbf{1 1}$, afforded thiourea $\mathbf{1 2}$. Oxidative cyclisation with bromine gave the target 5-fluoroaminobenzothiazole 14. In our hands, this cyclisation was the most efficient procedure. We investigated the use of the Johnson and Johnson protocol $^{28}$ but this resulted in the formation of complex mixtures.

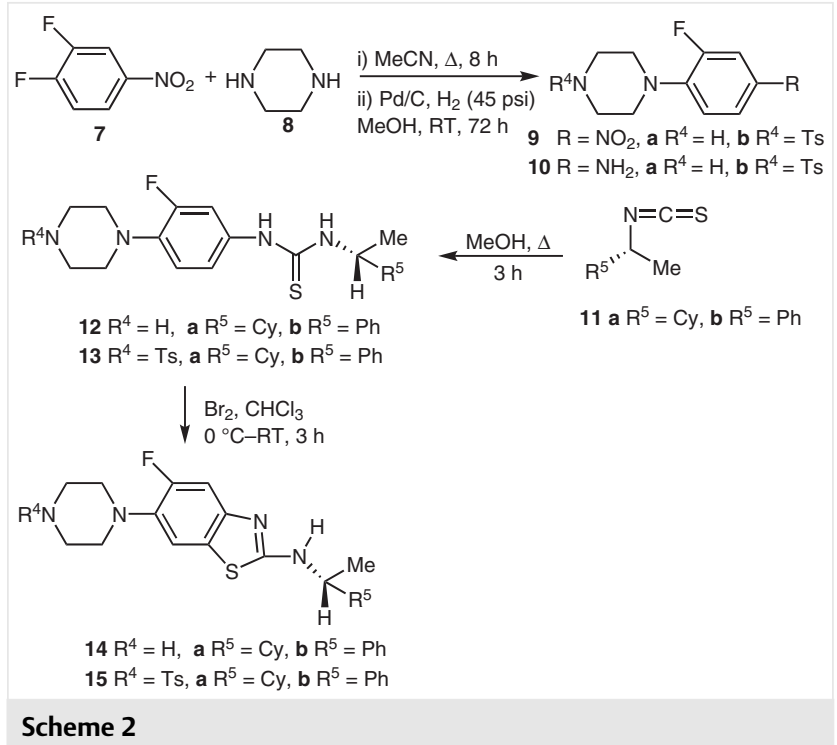

Having prepared these compounds, we proceeded to synthesise piperidine, pyrrolidine, and morpholino analogues (Scheme 3 and Figure 3), by employing a similar protocol to that used for the preparation of $\mathbf{1 4}$ and $\mathbf{1 5 .}$

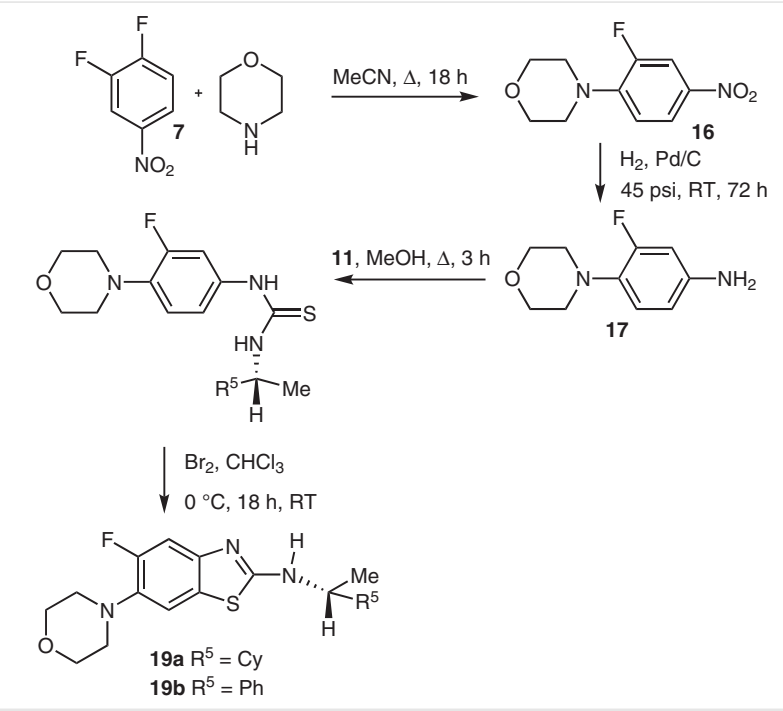

Scheme 3 


\section{Synopen}<smiles>O=[N+]([O-])c1ccc(N2CCCCC2)c(F)c1</smiles><smiles>Nc1ccc(N2CCCCC2)c(F)c1</smiles><smiles>O=[N+]([O-])c1ccc(N2CCCC2)c(F)c1</smiles><smiles>Nc1ccc(N2CCCC2)c(F)c1</smiles><smiles>[X]c1ccc(NC(=S)NC([Y6])([Y])[2H])cc1F</smiles>

22a $X=P i p, Y=C y$ 22b $X=P i p, Y=P h$ 22c $X=P y r r o, ~ Y=C y$ 22d $X=$ Pyrro, $Y=P h$ 13a $X=P i p T s, Y=C Y$ 13b X $=$ PipTs, $Y=P h$<smiles>[X]c1cc2sc(NC([Y6])([Y])[3H])nc2cc1F</smiles>

Figure 3

All of the prepared compounds were screened for antiTB activity using a broth dilution assay using $M$. tuberculosis strain H37Rv. Benzothiazoles exhibited MIC values in the range of $5-100 \mu \mathrm{g} / \mathrm{mL}$ whilst the precursor thioureas did not show any inhibition at concentrations of $100 \mu \mathrm{g} / \mathrm{mL}$.

In summary, we have prepared a series of chiral thioureas and 5-fluoroaminobenzothiazoles by employing a straightforward methodology.

Column chromatography was carried out using Merck 230-400 mesh silica gel. TLC was run on precoated silica gel 60 F254 plates. Specific rotations were measured with a Beltingham \& Stanley Ltd. ADP 200 polarimeter at $\lambda=589 \mathrm{~nm}$. All NMR spectra were recorded with a Bruker 600,400 or $300 \mathrm{MHz}$ spectrometer in the deuterated solvents stated. Anhydrous reactions were performed under argon or nitrogen atmospheres.

\section{1-(2-Fluoro-4-nitrophenyl)piperazine (9) $)^{29}$}

3,4-Difluoronitrobenzene $\mathbf{7}$ ( $1.43 \mathrm{~g}, 8.98 \mathrm{mmol}$ ) was dissolved in acetonitrile $(30 \mathrm{~mL})$. Piperazine $8(2.00 \mathrm{~g}, 22.48 \mathrm{mmol})$ was then added and the mixture was heated to reflux for $8 \mathrm{~h}$. The solution was cooled to ambient temperature and filtered. The filtrate was concentrated in vacuo to afford an orange solid, subsequent chromatographic purification with chloroform and $\mathrm{MeOH}$ ( 7:3) as eluents afforded 9.

Yield: $1.97 \mathrm{~g}$ (98\%); yellow solid; mp 68-69 ${ }^{\circ} \mathrm{C}\left[\right.$ lit. $\left.^{29} 68.5-71^{\circ} \mathrm{C}\right]$.
IR (thin film): $3228(\mathrm{NH}), 2911,2849,1602(\mathrm{Ar}), 1497\left(\mathrm{NO}_{2}\right), 1453$, 1389, $1323\left(\mathrm{NO}_{2}\right), 1263,1237$ (CF), 1203, 1155, 1144, 951, 936, 880, $846,815,802,745,709 \mathrm{~cm}^{-1}$.

${ }^{1} \mathrm{H}$ NMR $\left(300 \mathrm{MHz}, \mathrm{CDCl}_{3}\right): \delta=7.9(\mathrm{dd}, J=2.5,9.0 \mathrm{~Hz}, 1 \mathrm{H}), 7.9(\mathrm{dd}, J=$ 2.5, $13.2 \mathrm{~Hz}, 1 \mathrm{H}), 6.9$ (t, J = 8.8 Hz, $1 \mathrm{H}), 3.3$ (m, $4 \mathrm{H}), 3.0$ (m, $4 \mathrm{H}), 1.9$ (s, br, $1 \mathrm{H})$.

${ }^{13} \mathrm{C}$ NMR $\left(100 \mathrm{MHz}, \mathrm{CDCl}_{3}\right): \delta=153.0\left(J_{\mathrm{C}-\mathrm{F}}=249.3 \mathrm{~Hz}\right), 146.0\left(\mathrm{~J}_{\mathrm{C}-\mathrm{F}}=\right.$ $7.5 \mathrm{~Hz}), 140.4\left(J_{\mathrm{C}-\mathrm{F}}=9.4 \mathrm{~Hz}\right), 121.0\left(J_{\mathrm{C}-\mathrm{F}}=2.9 \mathrm{~Hz}\right), 117.0\left(J_{\mathrm{C}-\mathrm{F}}=4.0 \mathrm{~Hz}\right)$, $112.5\left(J_{\mathrm{C}-\mathrm{F}}=26.4 \mathrm{~Hz}\right), 50.8\left(J_{\mathrm{C}-\mathrm{F}}=5.0 \mathrm{~Hz}\right), 45.9$.

$\mathrm{UV}: \lambda_{\max }(\mathrm{MeOH}): 368(\varepsilon=7648) \mathrm{nm}$.

HRMS: $m / z$ calcd for $\mathrm{C}_{10} \mathrm{H}_{12} \mathrm{FN}_{3} \mathrm{O}_{2} \mathrm{Na}$ : 248.0811; found: 248.0806 .

\section{3-Fluoro-4-(piperazin-1-yl)aniline (10)}

The nitro compound $\mathbf{9}$ (4.00 g, $17.80 \mathrm{mmol}$ ) was dissolved in anhydrous $\mathrm{MeOH}(40 \mathrm{~mL})$. The resultant solution was hydrogenated at $45-$ 50 psi using $\mathrm{Pd} / \mathrm{C}(0.4 \mathrm{~g})$ as catalyst at r.t., for $72 \mathrm{~h}$. Note: The addition of the catalyst was conducted under a nitrogen atmosphere and subsequently evacuated $(3 x)$ and replaced with a hydrogen atmosphere. The reaction mixture was filtered through a plug of Celite and washed with $\mathrm{MeOH}(100 \mathrm{~mL})$. Subsequent removal of the solvent in vacuo afforded $\mathbf{1 0}$.

Yield: $3.00 \mathrm{~g}$ (86\%); brown solid; $\mathrm{mp} 188-190{ }^{\circ} \mathrm{C}$.

IR (thin film): 3337 (NH), $3190(\mathrm{NH}), 2948,2832,1656,1629$ (Ar), 1513, 1455, 1304, 1318, 1266, 1233 (CF), 1220, 1137, 1061, 969, 933 , $871,833,821,807,743 \mathrm{~cm}^{-1}$.

${ }^{1} \mathrm{H}$ NMR $\left(600 \mathrm{MHz}, \mathrm{CDCl}_{3}\right): \delta=6.8(\mathrm{~m}, 1 \mathrm{H}), 6.4(\mathrm{dd}, J=2.2,14.3 \mathrm{~Hz}$, $1 \mathrm{H}), 6.3$ (dd, J = 2.3, $8.5 \mathrm{~Hz}, 1 \mathrm{H}), 5.3(\mathrm{NH}, \mathrm{s}, 2 \mathrm{H}, \mathrm{br}), 3.5(\mathrm{~m}, 4 \mathrm{H}), 2.8$ (m, $4 \mathrm{H}), 1.9$ (s, br, $1 \mathrm{H})$.

${ }^{13} \mathrm{C} \mathrm{NMR}\left(150 \mathrm{MHz}, \mathrm{CDCl}_{3}\right): \delta=156.3\left(J_{\mathrm{C}-\mathrm{F}}=242.3 \mathrm{~Hz}\right), 145.2\left(J_{\mathrm{C}-\mathrm{F}}=\right.$ $37.6 \mathrm{~Hz}), 129.0\left(J_{\mathrm{C}-\mathrm{F}}=10.3 \mathrm{~Hz}\right), 121.3\left(J_{\mathrm{C}-\mathrm{F}}=4.2 \mathrm{~Hz}\right), 109.8,102.0\left(J_{\mathrm{C}-\mathrm{F}}=\right.$ $23.3 \mathrm{~Hz}), 51.5\left(J_{\mathrm{C}-\mathrm{F}}=62.2 \mathrm{~Hz}\right), 45.3$.

UV: $\lambda_{\max }(\mathrm{MeOH}): 207(\varepsilon=11240) \mathrm{nm}$.

\section{(R)-1-(1-Cyclohexylethyl)-3-(3-fluoro-4-(piperazin-1-yl)phe-} nyl)thiourea (12a)

The isothiocyanate 11a $(0.47 \mathrm{~g}, 2.80 \mathrm{mmol})$ was dissolved in anhydrous $\mathrm{MeOH}(20 \mathrm{~mL})$ and an equimolar quantity of aromatic primary amine $10(0.55 \mathrm{~g}, 2.80 \mathrm{mmol})$ was added with stirring. The reaction mixture was heated to reflux at $65^{\circ} \mathrm{C}$ for $3 \mathrm{~h}$. The reaction was monitored by TLC using petroleum ether and EtOAc (1:1) as eluent. The solvent was evaporated to afford a light-brown solid. Additional purification via silica gel chromatography using petroleum ether and EtOAc (1:1) gave 12a.

Yield: $0.36 \mathrm{~g}$ (36\%); buff coloured solid; $\mathrm{mp} 160-162{ }^{\circ} \mathrm{C} ;[\alpha]_{\mathrm{D}}+13.8(\mathrm{c}$ $\left.0.3, \mathrm{CHCl}_{3}\right)$.

IR (thin film): 3292 (NH), 3197, 2936, 2854, 1729, 1626 (Ar), 1538, 1513, 1446, 1409, 1364, 1343, 1305 (CS), 1280, 1242 (CF) $\mathrm{cm}^{-1}$.

${ }^{1} \mathrm{H}$ NMR $\left(600 \mathrm{MHz}, \mathrm{CDCl}_{3}\right): \delta=6.8(\mathrm{t}, J=8.9 \mathrm{~Hz}, 1 \mathrm{H}), 6.4(\mathrm{dd}, J=2.41$, $13.4 \mathrm{~Hz}, 1 \mathrm{H}), 6.4(\mathrm{dd}, J=2.4,8.4 \mathrm{~Hz}, 1 \mathrm{H}), 5.4(\mathrm{~d}, J=8.3 \mathrm{~Hz}, \mathrm{NH}), 4.6$ $(\mathrm{m}, J=6.7,7.7,6.7 \mathrm{~Hz}, 1 \mathrm{H}), 3.9(\mathrm{~m}, 4 \mathrm{H}), 3.6(\mathrm{~s}, \mathrm{br}, \mathrm{NH}), 3.0(\mathrm{~m}$, $4 \mathrm{H}), 1.8$ (s, br, $1 \mathrm{H}), 1.6-1.1(\mathrm{~m}, 14 \mathrm{H})$.

${ }^{13} \mathrm{C}$ NMR $\left(150 \mathrm{MHz}, \mathrm{CDCl}_{3}\right): \delta=181.8,156.9\left(J_{\mathrm{C}-\mathrm{F}}=245.3 \mathrm{~Hz}\right), 143.3$ $\left(J_{C-F}=10.5 \mathrm{~Hz}\right), 130.9\left(J_{C-F}=9.9 \mathrm{~Hz}\right), 120.8\left(J_{C-F}=4.1 \mathrm{~Hz}\right), 110.7\left(J_{C-F}=\right.$ $2.9 \mathrm{~Hz}), 103.8\left(J_{\mathrm{C}-\mathrm{F}}=23.7 \mathrm{~Hz}\right), 55.8,50.9,47.7,43.1,29.6,26.5,26.3$, 17.5.

HRMS: $m / z$ calcd for $\mathrm{C}_{19} \mathrm{H}_{30} \mathrm{FN}_{4} \mathrm{~S}$ : 365.2170; found: 365.2168 . 
(R)- $N$-(1-Cyclohexylethyl)-5-fluoro-6-(piperazin-1-yl)benzo[d]thiazol-2-amine (14a)

The thiourea 12a $(0.10 \mathrm{~g}, 0.27 \mathrm{mmol})$ was dissolved in chloroform (15 $\mathrm{mL}$ ) and cooled to $0{ }^{\circ} \mathrm{C}$. To this, an equimolar quantity of $\mathrm{Br}_{2}(0.01 \mathrm{~mL})$ was added. From this stock solution, $2 \mathrm{~mL}$ of solution was added to the reaction mixture. Following addition, the ice bath was removed and the reaction mixture was warmed to r.t. The mixture was stirred for a further $3 \mathrm{~h}$ and the resulting mixture was neutralized with saturated $\mathrm{NaHCO}_{3}$ and extracted with $\mathrm{CH}_{2} \mathrm{Cl}_{2}(3 \times 10 \mathrm{~mL})$. The combined organic phases were dried over $\mathrm{Na}_{2} \mathrm{SO}_{4}$ and concentrated in vacuo. The resulting residue was purified by chromatography on silica, using petroleum ether and EtOAc (1:1) as eluent. Removal of the solvent in vacuo afforded the product 14a.

Yield: $16 \mathrm{mg}$ (16\%); light-brown amorphous solid; $[\alpha]_{\mathrm{D}}-29.6$ (c 0.14 , $\mathrm{CHCl}_{3}$ ).

IR (thin film): 3342 (NH), 2924, 2852, 1708, 1625 (Ar), 1507, 1449 (CN thiazole), 1361 (CN thiazole), 1256 (CF), 1220, 1163, 999, 864, $745 \mathrm{~cm}^{-1}$.

${ }^{1} \mathrm{H}$ NMR $\left(600 \mathrm{MHz}, \mathrm{CDCl}_{3}\right): \delta=7.0(\mathrm{~d}, J=8.5 \mathrm{~Hz}, 1 \mathrm{H}), 6.5(\mathrm{~d}, J=$ $12.9 \mathrm{~Hz}, 1 \mathrm{H}), 4.2(\mathrm{~d}, J=8.4 \mathrm{~Hz}, 1 \mathrm{H}, \mathrm{NH}), 3.7(\mathrm{~m}, J=6.6,8.0,6.6 \mathrm{~Hz}$, $1 \mathrm{H}), 3.5(\mathrm{~m}, 4 \mathrm{H}), 2.9(\mathrm{t}, J=5.0 \mathrm{~Hz}, 4 \mathrm{H}), 1.75(\mathrm{~s}, 1 \mathrm{H}, \mathrm{br}), 1.7-0.8(\mathrm{~m}$, $14 \mathrm{H})$.

${ }^{13} \mathrm{C} \mathrm{NMR}\left(150 \mathrm{MHz}, \mathrm{CDCl}_{3}\right): \delta=170.0,157.1\left(\mathrm{~J}_{\mathrm{C}-\mathrm{F}}=64.8 \mathrm{~Hz}\right), 140.3\left(J_{\mathrm{C}-\mathrm{F}}=\right.$ $10.2 \mathrm{~Hz}), 132.3\left(J_{\mathrm{C}-\mathrm{F}}=11.0 \mathrm{~Hz}\right), 127.0,127.6,103.9\left(J_{\mathrm{C}-\mathrm{F}}=25.2 \mathrm{~Hz}\right)$, $51.1,50.6,44.0,43.4,29.3,28.9,26.5,18.5$.

UV: $\lambda_{\max }(\mathrm{MeOH})=210.9(\varepsilon=29368) \mathrm{nm}$.

\section{(R)-(-)-(1-Isothiocyanatoethyl)benzene (11b) ${ }^{30}$}

$1,1^{\prime}$-Thiocarbonyldiimidazole $(0.14 \mathrm{~g}, 0.82 \mathrm{mmol})$ was added to a rapidly stirring mixture of $\mathrm{CH}_{2} \mathrm{Cl}_{2}(18 \mathrm{~mL})$ and water $(18 \mathrm{~mL})$ at r.t., then $(R)-(+)-\alpha$-methylbenzylamine $(0.10 \mathrm{~g}, 0.82 \mathrm{mmol})$ was added. $\mathrm{NaH}-$ $\mathrm{CO}_{3}(0.18 \mathrm{~g}, 2.2 \mathrm{mmol})$ was then added to the resultant mixture slowly over a period of $45 \mathrm{~min}$, and stirring was continued at r.t. for $13 \mathrm{~h}$. The two phases were separated and the organic phase was dried over $\mathrm{Na}_{2} \mathrm{SO}_{4}$. The solvent was removed in vacuo to afford the isothiocyanate as a light-brown semisolid, which was further purified by column chromatography using petroleum ether and EtOAc (10:1) to afford the title compound $\mathbf{1 1 b}$.

Yield: $0.06 \mathrm{~g}(45 \%)$; light-brown oil; $[\alpha]_{\mathrm{D}}-13.2$ (c 3.0, $\left.\mathrm{CHCl}_{3}\right)\left\{\right.$ lit. $^{30 \mathrm{a}}$ $[\alpha]_{D}-4.3$ (c 1.0, acetone); lit. $\left.{ }^{30 \mathrm{~b}}[\alpha]_{\mathrm{D}}-17.3\left(\mathrm{CHCl}_{3}\right)\right\}$.

IR (thin film): 3033, 2985, 2933, 2085 (NCS), 1736, 1604 (Ar), 1494, 1454, 1374, 1345, 1326, 1308, 1278, 1242, 1203, 1182, 1158, 1103, $1067,1045 \mathrm{~cm}^{-1}$.

${ }^{1} \mathrm{H} \mathrm{NMR}\left(600 \mathrm{MHz}, \mathrm{CDCl}_{3}\right): \delta=7.3(\mathrm{~m}, 2 \mathrm{H}), 7.3(\mathrm{t}, J=6.8 \mathrm{~Hz}, 3 \mathrm{H}), 4.9$ $(\mathrm{q}, J=6.7,6.7,6.8 \mathrm{~Hz}, 1 \mathrm{H}), 1.6(\mathrm{~d}, J=6.8 \mathrm{~Hz}, 3 \mathrm{H})$.

${ }^{13} \mathrm{C}$ NMR $\left(150 \mathrm{MHz}, \mathrm{CDCl}_{3}\right): \delta=140.0,132.1,128.8,128.1,125.3,56.9$, 24.9.

UV: $\lambda_{\max }(\mathrm{MeOH})=206(\varepsilon=15129) \mathrm{nm}$.

(R)-1-(3-Fluoro-4-(piperazin-1-yl)phenyl)-3-(1-phenylethyl)thiourea (12b)

The isothiocyanate 11b $(0.51 \mathrm{~g}, 3.00 \mathrm{mmol})$ was dissolved in anhydrous $\mathrm{MeOH}(15 \mathrm{~mL})$ and an equimolar quantity of primary amine $\mathbf{1 0}$ was added with stirring. The reaction mixture was heated to reflux for $2 \mathrm{~h}$ at $65^{\circ} \mathrm{C}$. On completion of the reaction, the resultant mixture was concentrated in vacuo to afford a creamish white solid. Chromatographic purification (petroleum ether and EtOAc (1:1)) gave the product $\mathbf{1 2 b}$.
Yield: $0.30 \mathrm{~g}$ (28\%); colorless solid; $\mathrm{mp} 126-128{ }^{\circ} \mathrm{C} ;[\alpha]_{\mathrm{D}}-20.8(\mathrm{c} 0.8$, $\mathrm{CHCl}_{3}$ ).

IR (thin film): 3342 (NH), 2826, 1630 (Ar), 1513, 1339 (CS), 1280, 1219 (CF), 1014, 922, $808 \mathrm{~cm}^{-1}$.

${ }^{1} \mathrm{H} \mathrm{NMR}\left(400 \mathrm{MHz}, \mathrm{CDCl}_{3}\right): \delta=7.3(\mathrm{~m}, 5 \mathrm{H}), 6.7(\mathrm{t}, J=9.0 \mathrm{~Hz}, 1 \mathrm{H}), 6.4$ (m, $2 \mathrm{H}), 5.8$ (p, J = 6.9, 7.0, 7.0, $6.8 \mathrm{~Hz}, 1 \mathrm{H}), 5.7$ (d, $J=7.5 \mathrm{~Hz}, 1 \mathrm{H}, \mathrm{NH})$, $3.1(\mathrm{~s}, \mathrm{br}, \mathrm{NH}), 3.9(\mathrm{~m}, 4 \mathrm{H}), 2.0(\mathrm{~s}, \mathrm{br}, 1 \mathrm{H}), 2.9(\mathrm{~m}, 4 \mathrm{H}), 1.6(\mathrm{~d}, J=$ $6.8 \mathrm{~Hz}, 3 \mathrm{H})$.

13C NMR (100 MHz, CDCl3): $\delta=181.4,153.5(\mathrm{JC}-\mathrm{F}=43.5 \mathrm{~Hz}), 143.3$ $(\mathrm{JC}-\mathrm{F}=10.4 \mathrm{~Hz}), 143.0,130.8\left(J_{\mathrm{C}-\mathrm{F}}=10.2 \mathrm{~Hz}\right), 128.7,127.4,126.4$, $120.8\left(J_{C-F}=4.1 \mathrm{~Hz}\right), 110.6\left(J_{C-F}=3.0 \mathrm{~Hz}\right), 103.8\left(J_{C-F}=23.6 \mathrm{~Hz}\right), 54.6$, $50.8\left(J_{\mathrm{C}-\mathrm{F}}=2.0 \mathrm{~Hz}\right), 47.7,21.5$.

UV: $\lambda_{\max }(\mathrm{MeOH})=206(\varepsilon=50716) \mathrm{nm}$.

HRMS: $m / z$ calcd for $\mathrm{C}_{19} \mathrm{H}_{22} \mathrm{FN}_{4} \mathrm{~S}$ : 357.1544; found: 357.1510 .

\section{(R)-5-Fluoro- $N$-(1-phenylethyl)-6-(piperazin-1-yl)benzo[d] thi-} azol-2-amine (14b)

Thiourea 12b ( $0.10 \mathrm{~g}, 0.28 \mathrm{mmol})$ was dissolved in chloroform (15 $\mathrm{mL}$ ) and the reaction mixture was cooled in an ice bath. Subsequently, an equimolar quantity of $\mathrm{Br}_{2}(0.014 \mathrm{~mL})$ was added, the ice bath was removed, and the reaction mixture was brought to r.t. Stirring was continued for a further $15 \mathrm{~h}$, then the mixture was neutralized with saturated $\mathrm{NaHCO}_{3}$ and extracted with $\mathrm{CH}_{2} \mathrm{Cl}_{2}(3 \times 10 \mathrm{~mL})$. The combined organic phases were dried over $\mathrm{Na}_{2} \mathrm{SO}_{4}$ and concentrated in vacuo. Chromatography on silica eluting with petroleum ether and EtOAc ( $7: 3)$ with $1 \%$ triethylamine as the eluent gave benzothiazole 14b.

Yield: $22 \mathrm{mg}$ (21\%); brown amorphous solid; $[\alpha]_{\mathrm{D}}-66.7$ (c 0.2, $\mathrm{CHCl}_{3}$ ). IR (thin film): 3340, 1627 (Ar), 1508, 1451 (CN thiazole), 1418, 1386 (CN thiazole), 1257 (CF), $1222 \mathrm{~cm}^{-1}$.

${ }^{1} \mathrm{H} \mathrm{NMR}\left(600 \mathrm{MHz}, \mathrm{CDCl}_{3}\right): \delta=7.3(\mathrm{~m}, 5 \mathrm{H}), 6.9(\mathrm{~d}, J=8.5 \mathrm{~Hz}, 1 \mathrm{H}), 6.5$ $(\mathrm{d}, J=13.0 \mathrm{~Hz}, 1 \mathrm{H}), 5.0(\mathrm{q}, J=6.9,6.9,6.9 \mathrm{~Hz}, 1 \mathrm{H}), 4.6(\mathrm{~d}, J=6.9 \mathrm{~Hz}$, $\mathrm{NH}), 3.5(\mathrm{~m}, 4 \mathrm{H}), 2.9(\mathrm{t}, J=5.0 \mathrm{~Hz}, 4 \mathrm{H}), 1.6(\mathrm{~s}, \mathrm{br}, \mathrm{NH}), 1.5(\mathrm{~d}, J=$ $6.8 \mathrm{~Hz}, 3 \mathrm{H})$.

${ }^{13} \mathrm{C}$ NMR $\left(150 \mathrm{MHz}, \mathrm{CDCl}_{3}\right): \delta=174.9,156.8,144.2,140.4\left(J_{\mathrm{C}-\mathrm{F}}=\right.$ $11.0 \mathrm{~Hz}), 132.2\left(J_{\mathrm{C}-\mathrm{F}}=37.3 \mathrm{~Hz}\right), 128.6,127.7,126.1,123.7\left(J_{\mathrm{C}-\mathrm{F}}=\right.$ $3.9 \mathrm{~Hz}), 103.9\left(J_{\mathrm{C}-\mathrm{F}}=25.2 \mathrm{~Hz}\right), 103.1,51.0,50.2,44.0,22.5$.

HRMS: $m / z$ calcd for $\mathrm{C}_{19} \mathrm{H}_{23} \mathrm{FN}_{4} \mathrm{~S}$ : 357.1544 ; found: 357.1483 .

\section{1-(2-Fluoro-4-nitrophenyl)-4-tosylpiperazine (9b)}

The piperazine substituted nitro aromatic $\mathbf{8}(2.10 \mathrm{~g}, 9.32 \mathrm{mmol})$ was dissolved in $\mathrm{CH}_{2} \mathrm{Cl}_{2}(20 \mathrm{~mL})$. Triethylamine $(1.30 \mathrm{~mL}, 9.32 \mathrm{mmol})$ and p-toluenesulfonyl chloride ( $1.95 \mathrm{~g}, 10.26 \mathrm{mmol}$ ) were added and the mixture was allowed to stir at r.t. for $3.5 \mathrm{~h}$. The residue was filtered and the filtrate was evaporated in vacuo. The resulting residue was purified by chromatography using petroleum ether and EtOAc (3:1) as the eluent to afford $\mathbf{9 b}$.

Yield: $3.02 \mathrm{~g}$ (86\%); pale-yellow solid; $\mathrm{mp} 164-166{ }^{\circ} \mathrm{C}$.

IR (thin film): 3076, 2858, 1670, 1601, $1497\left(\mathrm{NO}_{2}\right), 1445,1391,1336$ $\left(\mathrm{NO}_{2}\right), 1281,1235(\mathrm{CF}), 1213,1157\left(\mathrm{SO}_{2}\right), 1114,1079,945,926,885$, $805 \mathrm{~cm}^{-1}$.

${ }^{1} \mathrm{H} \mathrm{NMR}\left(400 \mathrm{MHz}, \mathrm{CDCl}_{3}\right): \delta=7.9(\mathrm{dd}, J=2.4,8.9 \mathrm{~Hz}, 1 \mathrm{H}), 7.8(\mathrm{dd}, J=$ 2.5, $12.8 \mathrm{~Hz}, 1 \mathrm{H}), 7.7(\mathrm{~d}, J=8.1 \mathrm{~Hz}, 2 \mathrm{H}), 7.3(\mathrm{~d}, J=8.1 \mathrm{~Hz}, 2 \mathrm{H}), 6.9(\mathrm{t}$, $J=8.7 \mathrm{~Hz}, 1 \mathrm{H}), 3.3(\mathrm{~m}, 4 \mathrm{H}), 3.2(\mathrm{~m}, 4 \mathrm{H}), 2.4(\mathrm{~s}, 3 \mathrm{H})$.

${ }^{13} \mathrm{C} \mathrm{NMR}\left(150 \mathrm{MHz}, \mathrm{CDCl}_{3}\right): \delta=153.2\left(J_{\mathrm{C}-\mathrm{F}}=249.5 \mathrm{~Hz}\right), 144.8\left(J_{\mathrm{C}-\mathrm{F}}=\right.$ $8.0 \mathrm{~Hz}), 144.1,141.4\left(J_{\mathrm{C}-\mathrm{F}}=8.8 \mathrm{~Hz}\right), 132.2,129.9,127.9,120.9\left(J_{\mathrm{C}-\mathrm{F}}=\right.$ $3.2 \mathrm{~Hz}), 117.6\left(J_{\mathrm{C}-\mathrm{F}}=3.5 \mathrm{~Hz}\right), 112.6\left(J_{\mathrm{C}-\mathrm{F}}=26.3 \mathrm{~Hz}\right), 49.1\left(J_{\mathrm{C}-\mathrm{F}}=4.6 \mathrm{~Hz}\right)$, 45.8, 21.6. 
UV: $\lambda_{\max }(\mathrm{MeOH})=352(\varepsilon=20379) \mathrm{nm}$.

HRMS: $m / z$ calcd for $\mathrm{C}_{17} \mathrm{H}_{17} \mathrm{FN}_{3} \mathrm{O}_{4} \mathrm{~S}$ : 378.0918; found: 378.0876 .

\section{3-Fluoro-4-(4-tosylpiperazine-1-yl)aniline (10b)}

The piperazine substituted nitro aromatic $\mathbf{9 b}(4.00 \mathrm{~g}, 10.55 \mathrm{mmol})$ was dissolved in anhydrous $\mathrm{MeOH}(40 \mathrm{~mL})$. The reaction flask was charged with $10 \%$ palladium on carbon and hydrogenated at $45-50$ psi. for $72 \mathrm{~h}$. The reaction mixture was filtered through a plug of Celite and washed with $\mathrm{MeOH}(100 \mathrm{~mL})$. Subsequent removal of the solvent in vacuo afforded $\mathbf{1 0 b}$.

Yield: $3.52 \mathrm{~g}$ (96\%); brownish solid; $\mathrm{mp} 181-184^{\circ} \mathrm{C}$.

IR (thin film): $3434(\mathrm{NH}), 3363(\mathrm{NH}), 2923,1636,1598,1515,1452$, 1393, 1338, $1319\left(\mathrm{SO}_{2}\right), 1272,1229$ (CF), $1158\left(\mathrm{SO}_{2}\right), 1123,1090$, $1016,948,806,726 \mathrm{~cm}^{-1}$.

${ }^{1} \mathrm{H} \mathrm{NMR}\left(300 \mathrm{MHz}, \mathrm{CDCl}_{3}\right): \delta=7.6(\mathrm{~d}, J=8.2 \mathrm{~Hz}, 2 \mathrm{H}), 7.3(\mathrm{~d}, J=7.9 \mathrm{~Hz}$, $2 \mathrm{H}), 6.7(\mathrm{t}, J=8.8 \mathrm{~Hz}, 1 \mathrm{H}), 6.4(\mathrm{~m}, 2 \mathrm{H}), 3.5(\mathrm{NH}, \mathrm{s}, 2 \mathrm{H}), 3.1(\mathrm{~m}, 4 \mathrm{H})$, $3.0(\mathrm{~m}, 4 \mathrm{H}), 2.4(\mathrm{~s}, 3 \mathrm{H})$.

${ }^{13} \mathrm{C}$ NMR $\left(150 \mathrm{MHz}, \mathrm{CDCl}_{3}\right): \delta=155.4\left(J_{\mathrm{C}-\mathrm{F}}=158.4 \mathrm{~Hz}\right), 143.8,143.4$, 143.3, 132.3, 129.7, 127.9, $120.9\left(J_{C-F}=4.0 \mathrm{~Hz}\right), 110.6\left(J_{C-F}=3.0 \mathrm{~Hz}\right)$, $103.7\left(J_{C-F}=23.7 \mathrm{~Hz}\right), 50.8\left(J_{C-F}=2.0 \mathrm{~Hz}\right), 46.4,21.5$.

UV: $\lambda_{\max }(\mathrm{MeOH})=234(\varepsilon=13777) \mathrm{nm}$.

\section{(R)-1-(1-Cyclohexylethyl)-3-(3-fluoro-4-(4-tosylpiperazin-1-} yl))thiourea (13a)

The isothiocyanate 11a $(54.0 \mathrm{mg}, 3.21 \mathrm{mmol})$ was dissolved in anhydrous $\mathrm{MeOH}(20 \mathrm{~mL})$ and an equimolar quantity of $\mathbf{1 0 b}(111.8 \mathrm{mg}$, $3.21 \mathrm{mmol}$ ) was added with stirring. The reaction mixture was heated at $70{ }^{\circ} \mathrm{C}$ for $2 \mathrm{~h}$. On completion of the reaction, the solvent was evaporated in vacuo to gave a dark-brown solid that was purified by chromatography using petroleum ether and EtOAc (1:1) to give 13a.

Yield: $0.14 \mathrm{~g}$ (85\%); light-brown solid; mp $170-171{ }^{\circ} \mathrm{C} ;[\alpha]_{\mathrm{D}}-11.9(\mathrm{c}$ 1.0, $\mathrm{CHCl}_{3}$ ).

IR (thin film): 3396 (NH), 2925, 1726, 1598 (Ar), 1510, 1451, 1377, $1349\left(\mathrm{SO}_{2}\right), 1332,1306(\mathrm{CS}), 1264(\mathrm{CF}), 1164,1138\left(\mathrm{SO}_{2}\right), 1118,1019$, $947,865,730 \mathrm{~cm}^{-1}$.

${ }^{1} \mathrm{H} \mathrm{NMR}\left(600 \mathrm{MHz}, \mathrm{CDCl}_{3}\right): \delta=7.6(\mathrm{~d}, J=8.2 \mathrm{~Hz}, 2 \mathrm{H}), 7.3(\mathrm{~d}, J=8.0 \mathrm{~Hz}$, $2 \mathrm{H}), 6.7(\mathrm{t}, J=8.8 \mathrm{~Hz}, 1 \mathrm{H}), 6.4(\mathrm{~m}, 2 \mathrm{H}), 5.7(\mathrm{~s}, \mathrm{NH}), 4.1(\mathrm{q}, J=7.2,7.2$, $7.2 \mathrm{~Hz}, 1 \mathrm{H}), 3.6(\mathrm{~s}, \mathrm{NH}), 3.1(\mathrm{~m}, 4 \mathrm{H}), 3.0(\mathrm{~m}, 4 \mathrm{H}), 2.4(\mathrm{~s}, 3 \mathrm{H}), 0.8-1.8$ $(\mathrm{m}, 14 \mathrm{H})$.

${ }^{13} \mathrm{C}$ NMR $\left(150 \mathrm{MHz}, \mathrm{CDCl}_{3}\right): \delta=176.0,156.7\left(\mathrm{~J}_{\mathrm{C}-\mathrm{F}}=245.2 \mathrm{~Hz}\right), 143.8$, 142.6, 132.3, 130.8, 129.7, 127.9, 120.8, $110.6\left(J_{\mathrm{C}-\mathrm{F}}=2.9 \mathrm{~Hz}\right), 103.7$ $\left.U_{C-F}=23.8 \mathrm{~Hz}\right), 70.8,50.7,46.3,42.1,33.1,32.8,29.7,27.1,21.5$.

UV: $\lambda_{\max }(\mathrm{MeOH})=230(\varepsilon=25484) \mathrm{nm}$.

HRMS: $m / z$ calcd for $\mathrm{C}_{26} \mathrm{H}_{35} \mathrm{FN}_{4} \mathrm{NaO}_{2} \mathrm{~S}_{2}$ : 541.2083; found: 541.2099.

\section{(R)-N-(1-Cyclohexylethyl)-5-fluoro-6-(4-tosylpiperazin-1-yl)ben-} zo[d] thiazol-2-amine (15a)

The thiourea $13 \mathbf{a}(0.17 \mathrm{~g}, 0.32 \mathrm{mmol})$ was dissolved in chloroform (15 $\mathrm{mL}$ ) and the reaction mixture was cooled in an ice bath. Subsequently, an equimolar quantity of $\mathrm{Br}_{2}(0.02 \mathrm{~mL})$ in $\mathrm{CHCl}_{3}(4 \mathrm{~mL})$ was added, the ice bath was removed and the reaction mixture was brought to r.t. The mixture was stirred at r.t. for $3 \mathrm{~h}$. On completion of the reaction, the resulting residue was concentrated in vacuo and was subjected to column chromatography (petroleum ether and EtOAc (7:3)). Subsequent in vacuo evaporation, afforded $\mathbf{1 5 a}$.

Yield: $0.07 \mathrm{~g}(41 \%)$; solid; $[\alpha]_{\mathrm{D}}-114.3\left(\mathrm{c} 0.04, \mathrm{CHCl}_{3}\right)$.
IR (thin film): 3365 (NH), 2981, 2099, 1600, 1474 (CN thiazole), 1393 (CN thiazole), $1329\left(\mathrm{SO}_{2}\right), 1217(\mathrm{CF}), 1158\left(\mathrm{SO}_{2}\right), 1035,805,726 \mathrm{~cm}^{-1}$. ${ }^{1} \mathrm{H} \mathrm{NMR}\left(300 \mathrm{MHz}, \mathrm{CDCl}_{3}\right): \delta=7.6(\mathrm{~d}, J=8.2 \mathrm{~Hz}, 2 \mathrm{H}), 7.3(\mathrm{~d}, J=8.0 \mathrm{~Hz}$, $2 \mathrm{H}), 6.9(\mathrm{~d}, J=8.5 \mathrm{~Hz}, 1 \mathrm{H}), 6.4(\mathrm{~d}, J=13.0 \mathrm{~Hz}, 1 \mathrm{H}), 3.9(\mathrm{~s}, \mathrm{br}, \mathrm{NH}), 3.5$ $(\mathrm{m}, J=6.6,7.5,6.8 \mathrm{~Hz}, 1 \mathrm{H}), 3.1(\mathrm{~m}, 4 \mathrm{H}), 3.0(\mathrm{~m}, 4 \mathrm{H}), 2.4(\mathrm{~s}, 3 \mathrm{H}), 1.8-$ $0.8(\mathrm{~m}, 14 \mathrm{H})$.

${ }^{13} \mathrm{C}$ NMR $\left(150 \mathrm{MHz}, \mathrm{CDCl}_{3}\right): \delta=173.4,155.9\left(\mathrm{~J}_{\mathrm{C}-\mathrm{F}}=245.7 \mathrm{~Hz}\right), 143.8$, $140.6\left(J_{C-F}=10.6 \mathrm{~Hz}\right), 132.3,131.7,131.6,129.7,127.9,123.8,103.8$ $\left(J_{C-F}=25.4 \mathrm{~Hz}\right), 50.6\left(J_{C-F}=2.0 \mathrm{~Hz}\right), 50.5,46.2,43.7,29.1,28.9,26.5$, 26.3, 19.7.

$\mathrm{UV}: \lambda_{\max }(\mathrm{MeOH})=208(\varepsilon=35862) \mathrm{nm}$.

(R)-1-(3-Fluoro-4-(4-tosylpiperazin-1-yl)phenyl)-3-(1-phenylethyl)thiourea (13b)

The isothiocyanate 11b $(0.14 \mathrm{~g}, 0.86 \mathrm{mmol})$ was dissolved in anhydrous $\mathrm{MeOH}(10 \mathrm{~mL})$ and an equimolar quantity of aromatic amine $10 \mathbf{b}(0.30 \mathrm{~g})$ was added with stirring. The reaction mixture was heated for $2 \mathrm{~h}$ then allowed to cool and poured into cold water, resulting in precipitation of the thiourea. The solid product was washed with water and purified by recrystallization from EtOAc and a small amount of EtOH to obtain 13b.

Yield: $0.22 \mathrm{~g}(52 \%)$; brown solid; $\mathrm{mp} 89-92^{\circ} \mathrm{C}$; $[\alpha]_{\mathrm{D}}-313.0$ (c 0.2 , $\mathrm{CHCl}_{3}$ ).

IR (thin film): 3253 (NH), 3068, 3027, 2967, 1545, 1495, 1451, 1346 $\left(\mathrm{SO}_{2}\right), 1332,1278,1260$ (CF), 1206, $1163\left(\mathrm{SO}_{2}\right), 1118,1087,1011,949$, $815,759,696 \mathrm{~cm}^{-1}$.

${ }^{1} \mathrm{H} \mathrm{NMR}\left(600 \mathrm{MHz}, \mathrm{CDCl}_{3}\right): \delta=7.6(\mathrm{~d}, J=8.1 \mathrm{~Hz}, 2 \mathrm{H}), 7.3(\mathrm{~d}, J=8.0 \mathrm{~Hz}$, $2 \mathrm{H}), 7.2-7.0(\mathrm{~m}, 5 \mathrm{H}), 6.7(\mathrm{t}, J=8.9 \mathrm{~Hz}, 1 \mathrm{H}), 6.4(\mathrm{~m}, 2 \mathrm{H}), 5.3(\mathrm{~s}, \mathrm{br}$, $\mathrm{NH}), 4.8(\mathrm{~m}, 1 \mathrm{H}), 3.6(\mathrm{br}, \mathrm{NH}), 3.1(\mathrm{~m}, 4 \mathrm{H}), 3.0(\mathrm{~m}, 4 \mathrm{H}), 2.4(\mathrm{~s}, 3 \mathrm{H})$, $1.4(\mathrm{~d}, J=6.7 \mathrm{~Hz}, 3 \mathrm{H})$.

${ }^{13} \mathrm{C}$ NMR $\left(150 \mathrm{MHz}, \mathrm{CDCl}_{3}\right): \delta=177.9,156.7\left(J_{\mathrm{C}-\mathrm{F}}=245.0 \mathrm{~Hz}\right), 143.7$ (2C), 143.3, 132.4, 130.9, 129.7, $127.9(2 \mathrm{C}), 127.6,125.6,120.9\left(\mathrm{~J}_{\mathrm{C}-\mathrm{F}}=\right.$ $3.6 \mathrm{~Hz}), 110.6\left(J_{\mathrm{C}-\mathrm{F}}=2.8 \mathrm{~Hz}\right), 103.7\left(J_{\mathrm{C}-\mathrm{F}}=23.8 \mathrm{~Hz}\right), 54.2,50.7,46.3$, 29.7, 21.5.

UV: $\lambda_{\max }(\mathrm{MeOH})=231(\varepsilon=18750) \mathrm{nm}$.

HRMS: $m / z$ calcd for $\mathrm{C}_{26} \mathrm{H}_{28} \mathrm{FN}_{4} \mathrm{~S}_{2} \mathrm{O}_{2}$ : 511.1632; found: 511.1597 .

\section{(R)-5-Fluoro- $N$-(1-phenylethyl)-6-(4-tosylpiperazin-1-yl)ben- zo[d]thiazol-2-amine (15b)}

The thiourea 13b $(0.10 \mathrm{~g}, 0.20 \mathrm{mmol})$ was dissolved in chloroform (15 $\mathrm{mL}$ ) and the reaction mixture was cooled in an ice bath. Subsequently, an equimolar quantity of $\mathrm{Br}_{2}(0.01 \mathrm{~mL})$ in $\mathrm{CHCl}_{3}(2 \mathrm{~mL})$ was added to the reaction mixture. The ice bath was removed and the reaction mixture was brought to r.t., and stirred at r.t. for $1 \mathrm{~h}$. The resulting solution was neutralized with saturated $\mathrm{NaHCO}_{3}$ and extracted with $\mathrm{CH}_{2}$ $\mathrm{Cl}_{2}(3 \times 10 \mathrm{~mL})$. The combined organic phases were dried over $\mathrm{Na}_{2} \mathrm{SO}_{4}$ and concentrated in vacuo. The resulting residue was purified by chromatography with petroleum ether and EtOAc (1:1). The combined fractions, on evaporation, afforded $\mathbf{1 5 b}$.

Yield: $0.03 \mathrm{~g}$ (30\%); light-brown solid; $[\alpha]_{\mathrm{D}}+13.1$ ( c $0.3, \mathrm{CHCl}_{3}$ ).

IR (thin film): 3357 (NH), 2924, 2854, 1709, 1537, 1440, 1453 (CN thiazole), 1350 (CN thiazole), 1330, 1219 (CF), 1165, 1093, 948, 862, 731, $701 \mathrm{~cm}^{-1}$.

${ }^{1} \mathrm{H} \mathrm{NMR}\left(600 \mathrm{MHz}, \mathrm{CDCl}_{3}\right): \delta=7.6(\mathrm{~d}, J=8.2 \mathrm{~Hz}, 2 \mathrm{H}), 7.3(\mathrm{~d}, J=7.2 \mathrm{~Hz}$, $2 \mathrm{H}), 7.3(\mathrm{~m}, 5 \mathrm{H}), 7.1(\mathrm{~d}, J=12.8 \mathrm{~Hz}, 1 \mathrm{H}), 7.0(\mathrm{~d}, J=7.0 \mathrm{~Hz}, 1 \mathrm{H}), 6.0(\mathrm{~s}$, br, NH), 4.7 (q, $J=6.6,6.6,6.7 \mathrm{~Hz}, 1 \mathrm{H}), 3.1(\mathrm{~m}, 4 \mathrm{H}), 3.0(\mathrm{~d}, J=4.7 \mathrm{~Hz}$, $4 \mathrm{H}), 2.4(\mathrm{~s}, 3 \mathrm{H}), 1.6(\mathrm{~d}, J=6.8 \mathrm{~Hz}, 3 \mathrm{H})$. 
${ }^{13} \mathrm{C}$ NMR $\left(150 \mathrm{MHz}, \mathrm{CDCl}_{3}\right): \delta=167.5,155.4\left(J_{\mathrm{C}-\mathrm{F}}=241.8 \mathrm{~Hz}\right), 143.9$, 142.5, 134.7, 134.0, 133.7, 132.2, 129.8, 128.8, 127.9, 127.8, 127.7, $126.1,125.9,111.0\left(J_{C-F}=3.3 \mathrm{~Hz}\right), 106.5\left(J_{C-F}=23.9 \mathrm{~Hz}\right), 55.4,50.7\left(J_{C-F}=\right.$ $2.3 \mathrm{~Hz}), 46.3,23.6,21.5$.

UV: $\lambda_{\max }(\mathrm{MeOH})=370(\varepsilon=66751) \mathrm{nm}$.

\section{4-(2-Fluoro-4-nitrophenyl)morpholine (16)}

3,4-Difluoronitrobenzene $7(1.10 \mathrm{~mL}, 6.90 \mathrm{mmol})$ was dissolved in acetonitrile $(30 \mathrm{~mL})$. To the resultant mixture, morpholine $(1.50 \mathrm{~mL}$, $17.29 \mathrm{mmol}$ ) was added. The mixture was heated to reflux for $18 \mathrm{~h}$, then the resultant mixture was poured into cold water and the residue was filtered. The residue was recrystallized with aqueous $\mathrm{MeOH}$ to afford 16.

Yield: $1.09 \mathrm{~g}$ (70\%); yellow solid; mp $110-112^{\circ} \mathrm{C}$ [lit. $\left.{ }^{29} 111-112{ }^{\circ} \mathrm{C}\right]$.

IR (thin film): 3053, 2853, 1602 (Ar), 1516, $1493\left(-\mathrm{NO}_{2}\right), 1446,1362$, $1320\left(\mathrm{NO}_{2}\right), 1273,1261,1243$ (CF), 1160, 1124, 1091, 1072, 1050, $1028,949,916,859,848,815,804,746 \mathrm{~cm}^{-1}$.

${ }^{1} \mathrm{H} \mathrm{NMR}\left(300 \mathrm{MHz}, \mathrm{CDCl}_{3}\right): \delta=8.0(\mathrm{~m}, 1 \mathrm{H}), 7.9(\mathrm{dd}, J=2.6,13.1 \mathrm{~Hz}$, $1 \mathrm{H}), 6.9(\mathrm{t}, J=8.7 \mathrm{~Hz}, 1 \mathrm{H}), 3.9(\mathrm{~m}, 4 \mathrm{H}), 3.3(\mathrm{~m}, 4 \mathrm{H})$.

${ }^{13} \mathrm{C} \mathrm{NMR}\left(150 \mathrm{MHz}, \mathrm{CDCl}_{3}\right): \delta=153.2\left(J_{\mathrm{C}-\mathrm{F}}=359.0 \mathrm{~Hz}\right), 145.5\left(J_{\mathrm{C}-\mathrm{F}}=\right.$ $6.0 \mathrm{~Hz}), 140.9\left(J_{\mathrm{C}-\mathrm{F}}=11.0 \mathrm{~Hz}\right), 121.0\left(J_{\mathrm{C}-\mathrm{F}}=4.0 \mathrm{~Hz}\right), 116.9,112.7\left(J_{\mathrm{C}-\mathrm{F}}=\right.$ $28.0 \mathrm{~Hz}), 66.6,49.9\left(J_{\mathrm{C}-\mathrm{F}}=5.0 \mathrm{~Hz}\right)$.

UV: $\lambda_{\max }(\mathrm{MeOH})=360(\varepsilon=10535) \mathrm{nm}$.

HRMS: $m / z$ calcd for $\mathrm{C}_{10} \mathrm{H}_{11} \mathrm{FN}_{2} \mathrm{O}_{3} \mathrm{Na}$ : 249.0646; found: 249.0595 .

\section{3-Fluoro-4-morpholinoaniline (17) ${ }^{31}$}

The nitro compound $\mathbf{1 6}$ (1.00 g, $4.42 \mathrm{mmol})$ was dissolved in anhydrous $\mathrm{MeOH}(40 \mathrm{~mL})$ and the reaction flask was charged with $10 \%$ palladium on carbon at 45-50 psi. The resultant mixture was shaken for $72 \mathrm{~h}$ at r.t. The reaction mixture was filtered through a plug of Celite and washed with $\mathrm{MeOH}(100 \mathrm{~mL})$. Subsequent removal of the solvent in vacuo afforded 17.

Yield: $0.80 \mathrm{~g}$ (92\%); slightly pink solid; $\mathrm{mp} 116-117^{\circ} \mathrm{C}$.

IR (thin film): 3418 (NH), 3337 (NH), 3232, 2974, 2824, 1639, 1578, 1513, 1479, 1450, 1376, 1317, 1301, 1272, 1249 (CF), 1221, 1206, $1161,1109,815,741 \mathrm{~cm}^{-1}$.

${ }^{1} \mathrm{H}$ NMR $\left(600 \mathrm{MHz}, \mathrm{CDCl}_{3}\right): \delta=6.8(\mathrm{t}, J=8.6 \mathrm{~Hz}, 1 \mathrm{H}), 6.4(\mathrm{dd}, J=2.5$, $13.5 \mathrm{~Hz}, 1 \mathrm{H}), 6.4$ (dd, J = 7.9, $2.8 \mathrm{~Hz}, 1 \mathrm{H}), 3.8(\mathrm{~m}, 4 \mathrm{H}), 3.6$ (br, s, $2 \mathrm{H}$, $\mathrm{NH}), 2.9$ ( $\mathrm{m}, 4 \mathrm{H})$.

${ }^{13} \mathrm{C} \mathrm{NMR}\left(150 \mathrm{MHz}, \mathrm{CDCl}_{3}\right): \delta=157.0\left(J_{\mathrm{C}-\mathrm{F}}=245.0 \mathrm{~Hz}\right), 142.8\left(J_{\mathrm{C}-\mathrm{F}}=\right.$ $11.0 \mathrm{~Hz}), 131.8\left(J_{\mathrm{C}-\mathrm{F}}=9.0 \mathrm{~Hz}\right), 120.3\left(J_{\mathrm{C}-\mathrm{F}}=4.0 \mathrm{~Hz}\right), 110.6\left(J_{\mathrm{C}-\mathrm{F}}=\right.$ $3.0 \mathrm{~Hz}), 103.9\left(J_{\mathrm{C}-\mathrm{F}}=24.0 \mathrm{~Hz}\right), 67.3,51.8\left(J_{\mathrm{C}-\mathrm{F}}=2.0 \mathrm{~Hz}\right)$.

UV: $\lambda_{\max }(\mathrm{MeOH})=248(\varepsilon=24248) \mathrm{nm}$.

HRMS: $m / z$ calcd for $\mathrm{C}_{10} \mathrm{H}_{13} \mathrm{FN}_{2} \mathrm{ONa}$ : 219.0904; found: 219.0854 .

\section{(R)-1-(1-Cyclohexylethyl)-3-(3-fluoro-4-morpholinophe- nyl)thiourea (18a)}

The isothiocyanate 11a $(0.09 \mathrm{~g}, 0.53 \mathrm{mmol})$ was dissolved in anhydrous $\mathrm{MeOH}(20 \mathrm{~mL})$ and an equimolar quantity of aromatic amine $\mathbf{1 7}$ $(0.10 \mathrm{~g}, 0.53 \mathrm{mmol})$ was added with stirring. The reaction mixture was then heated at $65^{\circ} \mathrm{C}$ for $3 \mathrm{~h}$. The reaction was monitored by TLC. On completion of the reaction, the solvent was evaporated to obtain the crude product, which was purified by column chromatography with petroleum ether and EtOAc (1:1) as the eluent. The eluents were then evaporated to afford 18a.

Yield: $0.60 \mathrm{~g}$ (30\%); off-white solid; $\mathrm{mp} 128-131{ }^{\circ} \mathrm{C}$; $[\alpha]_{\mathrm{D}}+57.2$ (c 2.7, $\mathrm{CHCl}_{3}$ ).
IR (thin film): $3172(\mathrm{NH}), 2924,2853,1579,1514,1449,1378,1303$ (CS), 1254 (CF), 1223, 1209, 1162, 1047, 998, 892, 859, 817, 790, $727 \mathrm{~cm}^{-1}$.

${ }^{1} \mathrm{H}$ NMR $\left(600 \mathrm{MHz}, \mathrm{CDCl}_{3}\right): \delta=7.6(\mathrm{~s}, \mathrm{br}, \mathrm{NH}), 6.9(\mathrm{~d}, J=9.1 \mathrm{~Hz}, 1 \mathrm{H})$, $6.9(\mathrm{~m}, 1 \mathrm{H}), 6.9(\mathrm{~m}, 1 \mathrm{H}), 5.7(\mathrm{NH}), 4.4(\mathrm{~m}, 1 \mathrm{H}), 3.8(\mathrm{~m}, 4 \mathrm{H}), 3.1(\mathrm{~m}$, $4 \mathrm{H}), 0.9-1.8(\mathrm{~m}, 14 \mathrm{H})$.

${ }^{13} \mathrm{C}$ NMR (150 MHz, $\left.\mathrm{CDCl}_{3}\right): \delta=179.9,155.7\left(J_{\mathrm{C}-\mathrm{F}}=249.0 \mathrm{~Hz}\right), 139.4$ $\left(J_{\mathrm{C}-\mathrm{F}}=16.0 \mathrm{~Hz}\right), 130.0\left(J_{\mathrm{C}-\mathrm{F}}=8.0 \mathrm{~Hz}\right), 121.9,119.5,114.2\left(J_{\mathrm{C}-\mathrm{F}}=\right.$ $22.0 \mathrm{~Hz}), 66.9,55.9,50.7\left(J_{\mathrm{C}-\mathrm{F}}=3.0 \mathrm{~Hz}\right), 42.9,29.3,28.9,26.4,17.3$.

UV: $\lambda_{\max }(\mathrm{MeOH})=258(\varepsilon=65390) \mathrm{nm}$.

HRMS: $m / z$ calcd for $\mathrm{C}_{19} \mathrm{H}_{27} \mathrm{FN}_{3} \mathrm{OS}$ : 364.1859; found: 364.1850 .

\section{(R)-N-(1-Cyclohexylethyl)-5-fluoro-6-morpholinobenzo[d]thi-} azole-2-amine (19a)

Thiourea 18a $(0.20 \mathrm{~g}, 0.54 \mathrm{mmol})$ was dissolved in chloroform $(15 \mathrm{~mL})$ and the reaction mixture was cooled in an ice bath at $0{ }^{\circ} \mathrm{C}$. Subsequently, an equimolar quantity of $\mathrm{Br}_{2}(0.03 \mathrm{~mL})$, dissolved in chloroform $(6 \mathrm{~mL})$ was added to the reaction mixture. The ice bath was removed and the reaction mixture was brought to r.t. then stirred at r.t. for $18 \mathrm{~h}$ and the progress of the reaction was monitored by TLC. Chromatographic purification using petroleum ether and EtOAC (1:1), and removal of the eluents in vacuo gave 19a.

Yield: $97.8 \mathrm{mg}$ (49\%); light-brown amorphous solid; $[\alpha]_{D}-16.0$ (c 1.3, $\left.\mathrm{CHCl}_{3}\right)$.

IR (thin film): 3376 (NH), 3046, 2926, 2855, 1609, 1509, 1479 (CN thiazole), 1450, 1364 (CN thiazole), 1292, 1265 (CF), 1179, 1123, 1049, $856,823,722,669$.

${ }^{1} \mathrm{H}$ NMR $\left(600 \mathrm{MHz}, \mathrm{CDCl}_{3}\right): \delta=7.1(\mathrm{~d}, J=12.8 \mathrm{~Hz}, 1 \mathrm{H}), 7.1(\mathrm{~d}, J=$ $8.0 \mathrm{~Hz}, 1 \mathrm{H}), 4.6(\mathrm{~s}, \mathrm{br}, \mathrm{NH}), 3.8(\mathrm{~m}, 4 \mathrm{H}), 3.4(\mathrm{~m}, 1 \mathrm{H}), 3.0(\mathrm{~m}, 4 \mathrm{H}), 1.0-$ $1.9(\mathrm{~m}, 14 \mathrm{H})$.

${ }^{13} \mathrm{C}$ NMR $\left(150 \mathrm{MHz}, \mathrm{CDCl}_{3}\right): \delta=176.2,155.6\left(J_{\mathrm{C}-\mathrm{F}}=244.0 \mathrm{~Hz}\right), 146.9$ $\left(J_{\mathrm{C}-\mathrm{F}}=12.0 \mathrm{~Hz}\right), 135.4\left(J_{\mathrm{C}-\mathrm{F}}=11.0 \mathrm{~Hz}\right), 124.6,110.7,105.9\left(J_{\mathrm{C}-\mathrm{F}}=\right.$ $24.0 \mathrm{~Hz}), 67.1,57.7,51.7\left(J_{\mathrm{C}-\mathrm{F}}=2.0 \mathrm{~Hz}\right), 43.6,29.1,26.2,21.4,18.0$.

UV: $\lambda_{\max }(\mathrm{MeOH})=225(\varepsilon=23953) \mathrm{nm}$.

HRMS: $m / z$ calcd for $\mathrm{C}_{19} \mathrm{H}_{27} \mathrm{FN}_{3} \mathrm{OS}$ : 364.1859; found: 364.1891 .

\section{(R)-1-(3-Fluoro-4-morpholinophenyl)-3-(1-phenylethyl)thiourea (18b)}

Isothiocyanate $\mathbf{1 1 b}(0.62 \mathrm{~g}, 3.80 \mathrm{mmol})$ was dissolved in anhydrous $\mathrm{MeOH}(10 \mathrm{~mL})$ and an equimolar quantity of primary amine 17 (0.75 $\mathrm{g}, 3.80 \mathrm{mmol}$ ) was added with stirring. The reaction mixture was heated to reflux for $2 \mathrm{~h}$ at $65^{\circ} \mathrm{C}$, then cooled and poured into ice cold water, where thiourea was precipitated. The solid product was washed with water and was purified by column chromatography using petroleum ether and EtOAc (1:1) as the eluent. The eluents were then evaporated to afford $\mathbf{1 8 b}$.

Yield: $0.03 \mathrm{~g}$ (24\%); white solid; $\mathrm{mp} 116-118^{\circ} \mathrm{C} ;[\alpha]_{\mathrm{D}}-87.3$ (c 2.2, CH$\mathrm{Cl}_{3}$ ).

IR (thin film): 3240 (NH), 3010, 2857, 1581, 1514, 1449, 1375, 1343, 1305 (CS), 1254 (CF), 1223, 1212, 1155, 1113, 1069, 1023, 920, 859, $818,792,726$.

${ }^{1} \mathrm{H}$ NMR (600 MHz, $\left.\mathrm{CDCl}_{3}\right): \delta=7.4(\mathrm{~s}, \mathrm{br}, \mathrm{NH}), 7.3(\mathrm{~m}, 2 \mathrm{H}), 7.3(\mathrm{~m}$, $3 \mathrm{H}), 6.9(\mathrm{~m}, 3 \mathrm{H}), 6.1(\mathrm{~s}, \mathrm{br}, \mathrm{NH}), 5.6(\mathrm{~m}, 1 \mathrm{H}), 3.8(\mathrm{~m}, 4 \mathrm{H}), 3.0(\mathrm{~m}$, $4 \mathrm{H}), 1.5(\mathrm{~d}, J=6.8 \mathrm{~Hz}, 3 \mathrm{H})$.

${ }^{13} \mathrm{C}$ NMR $\left(150 \mathrm{MHz}, \mathrm{CDCl}_{3}\right): \delta=180.1,155.5\left(\mathrm{~J}_{\mathrm{C}-\mathrm{F}}=250.9 \mathrm{~Hz}\right), 142.1$, $141.6,139.3,128.8,127.7,126.2,121.8,119.4,114.4\left(J_{C-F}=22.6 \mathrm{~Hz}\right)$, 66.9, 54.4, 50.6 $\left(J_{C-F}=2.6 \mathrm{~Hz}\right), 21.5$. 
UV: $\lambda_{\max }(\mathrm{MeOH})=250(\varepsilon=20700) \mathrm{nm}$.

HRMS: $m / z$ calcd for $\mathrm{C}_{19} \mathrm{H}_{21} \mathrm{FN}_{3} \mathrm{OS}$ : 358.1389; found: 358.1377 .

\section{(R)-5-Fluoro-6-morpholino- $N$-(1-phenylethyl)benzo[d]thiazol-2- amine (19b)}

Thiourea 18b (0.08 g, $0.22 \mathrm{mmol})$ was dissolved in chloroform (15 $\mathrm{mL}$ ) and the reaction mixture was cooled in an ice bath. Subsequently, an equimolar quantity of $\mathrm{Br}_{2}(0.01 \mathrm{~mL})$ in $\mathrm{CHCl}_{3}(2 \mathrm{~mL})$ was added to the reaction mixture. The ice bath was removed and the reaction mixture was brought to r.t. The mixture was stirred at r.t. for $18 \mathrm{~h}$, then the resulting solution was neutralized with saturated $\mathrm{NaHCO}_{3}$ and extracted with $\mathrm{CH}_{2} \mathrm{Cl}_{2}(3 \times 10 \mathrm{~mL})$. The combined organic phases were dried over anhydrous $\mathrm{Na}_{2} \mathrm{SO}_{4}$ and concentrated in vacuo. The resulting residue was purified by chromatography using petroleum ether and EtOAc (7:3). The combined eluents on evaporation afforded semisolid 18b.

Yield: $21 \mathrm{mg}(29 \%) ;[\alpha]_{\mathrm{D}}+65.3\left(\mathrm{c} 0.3, \mathrm{CHCl}_{3}\right)$.

IR (thin film): 3213 (NH), 2968, 2857, 2824, 1709, 1615, 1574, 1535, 1494, 1449 (CN thiazole), 1418, 1376, 1361 (CN thiazole), 1305, 1274, 1260 (CF), 1217, 1160, 1116, 1090, 1030, 999, 863, $700 \mathrm{~cm}^{-1}$.

${ }^{1} \mathrm{H} \mathrm{NMR}\left(600 \mathrm{MHz}, \mathrm{CDCl}_{3}\right): \delta=7.3(\mathrm{~m}, 2 \mathrm{H}), 7.3(\mathrm{~m}, 2 \mathrm{H}), 7.2(\mathrm{~m}, 1 \mathrm{H})$, $7.2(\mathrm{~d}, J=12.9 \mathrm{~Hz}, 1 \mathrm{H}), 7.1(\mathrm{~d}, J=8.0 \mathrm{~Hz}, 1 \mathrm{H}), 6.2(\mathrm{~s}, \mathrm{br}, \mathrm{NH}), 4.7(\mathrm{q}, J=$ 6.6, 6.6, 6.7 Hz, $1 \mathrm{H}), 3.8(\mathrm{~m}, 4 \mathrm{H}), 3.0(\mathrm{~m}, 4 \mathrm{H}), 1.6(\mathrm{~d}, J=6.7 \mathrm{~Hz}, 3 \mathrm{H})$.

${ }^{13} \mathrm{C} \mathrm{NMR}\left(150 \mathrm{MHz}, \mathrm{CDCl}_{3}\right): \delta=167.4,155.7\left(\mathrm{~J}_{\mathrm{C}-\mathrm{F}}=242.0 \mathrm{~Hz}\right), 147.6$ $\left(J_{C-F}=13.0 \mathrm{~Hz}\right), 142.7,135.5\left(J_{C-F}=11.1 \mathrm{~Hz}\right), 128.8,127.7,126.1,125.9$, $110.5\left(J_{C-F}=3.5 \mathrm{~Hz}\right), 106.6\left(J_{C-F}=2.4 \mathrm{~Hz}\right), 67.0,55.4,51.7\left(J_{C-F}=\right.$ $2.2 \mathrm{~Hz}), 23.6$.

UV: $\lambda_{\max }(\mathrm{MeOH})=225(\varepsilon=12599) \mathrm{nm}$.

HRMS: $m / z$ calcd for $\mathrm{C}_{19} \mathrm{H}_{19} \mathrm{FN}_{3} \mathrm{OS}$ : 356.1227; found: 356.1198 .

\section{1-(2-Fluoro-4-nitrophenyl)piperidine (20) ${ }^{32}$}

3,4-Difluoronitrobenzene $7(0.50 \mathrm{~mL}, 4.5 \mathrm{mmol})$ was dissolved in EtOH $(20 \mathrm{~mL})$, piperidine $(1.15 \mathrm{~mL}, 11.6 \mathrm{mmol})$ was added and the mixture was heated to reflux for $8 \mathrm{~h}$. The solvent was removed in vacuo and the residue was re-dissolved in EtOAc $(20 \mathrm{~mL})$ and washed with water $(3 \times 20 \mathrm{~mL})$. The EtOAc layer was dried over $\mathrm{Na}_{2} \mathrm{SO}_{4}$, filtered and the solvent was removed in vacuo to afford $\mathbf{2 0}$.

Yield: $0.64 \mathrm{~g}$ (64\%); oil.

IR (thin film): 3088, 2937, 2854, 1601 (Ar), $1501\left(\mathrm{NO}_{2}\right), 1452,1322$ $\left(\mathrm{NO}_{2}\right), 1267,1239(\mathrm{CF}), 1256,1024,946,881,803,744 \mathrm{~cm}^{-1}$.

${ }^{1} \mathrm{H} \mathrm{NMR}\left(300 \mathrm{MHz}, \mathrm{CDCl}_{3}\right): \delta=7.9(\mathrm{dd}, J=2.5,8.9 \mathrm{~Hz}, 1 \mathrm{H}), 7.8(\mathrm{dd}, J=$ 2.5, $13.3 \mathrm{~Hz}, 1 \mathrm{H}), 6.9(\mathrm{t}, J=8.8 \mathrm{~Hz}, 1 \mathrm{H}), 3.2(\mathrm{~m}, 4 \mathrm{H}), 1.7(\mathrm{~m}, 6 \mathrm{H})$.

${ }^{13} \mathrm{C} \mathrm{NMR}\left(75 \mathrm{MHz}, \mathrm{CDCl}_{3}\right): \delta=152.8\left(J_{\mathrm{C}-\mathrm{F}}=246.3 \mathrm{~Hz}\right), 146.5\left(J_{\mathrm{C}-\mathrm{F}}=\right.$ $7.9 \mathrm{~Hz}), 139.8,121.0\left(J_{\mathrm{C}-\mathrm{F}}=2.8 \mathrm{~Hz}\right), 117.0\left(J_{\mathrm{C}-\mathrm{F}}=4.1 \mathrm{~Hz}\right), 112.5\left(J_{\mathrm{C}-\mathrm{F}}=\right.$ $26.5 \mathrm{~Hz}), 51.0\left(J_{\mathrm{C}-\mathrm{F}}=5.3 \mathrm{~Hz}\right), 25.8,24.1$.

UV: $\lambda_{\max }(\mathrm{MeOH})=205(\varepsilon=16760) \mathrm{nm}$.

HRMS: $m / z$ calcd for $\mathrm{C}_{11} \mathrm{H}_{13} \mathrm{FN}_{2} \mathrm{NaO}_{2}$ : 247.0853; found: 247.0805.

\section{3-Fluoro-4-(piperidin-1-yl)aniline (21) ${ }^{32}$}

The nitro compound 20 (1.02 g, $4.5 \mathrm{mmol})$ was dissolved in anhydrous $\mathrm{MeOH}(40 \mathrm{~mL})$ and the reaction flask was charged with $10 \%$ palladium on carbon and hydrogenated at $45-50$ psi. The resultant mixture was shaken at r.t. for $72 \mathrm{~h}$. The reaction mixture was filtered through a plug of Celite and washed with $\mathrm{MeOH}(100 \mathrm{~mL})$. Subsequent removal of the solvent in vacuo afforded 21.
Yield: $0.88 \mathrm{~g}$ (99\%); brownish oil.

IR (thin film): $3420(\mathrm{NH}), 3341(\mathrm{NH}), 3220,2932,2851,2803,1629$ (Ar), 1578, 1510, 1466, 1450, 1310, 1275, 1258 (CF), 1229, 1207, $1166,1028,962,838,721 \mathrm{~cm}^{-1}$.

${ }^{1} \mathrm{H}$ NMR (300 MHz, MeOD): $\delta=8.0(\mathrm{t}, J=7.0 \mathrm{~Hz}, 1 \mathrm{H}), 7.5(\mathrm{~d}, J=$ $11.8 \mathrm{~Hz}, 1 \mathrm{H}$ ), 7.4 (d, J = 7.5 Hz, $1 \mathrm{H}), 5.3$ (s, br, $2 \mathrm{H}, \mathrm{NH}), 3.7$ (s, $4 \mathrm{H}), 2.1$ (s, $4 \mathrm{H}), 1.8(\mathrm{~s}, 2 \mathrm{H})$.

${ }^{13} \mathrm{C} \mathrm{NMR}\left(75 \mathrm{MHz}, \mathrm{CDCl}_{3}\right): \delta=155.3\left(J_{\mathrm{C}-\mathrm{F}}=253.0 \mathrm{~Hz}\right), 137.2\left(\mathrm{~J}_{\mathrm{C}-\mathrm{F}}=\right.$ $10.6 \mathrm{~Hz}), 127.5,124.6,119.3,111.5\left(J_{C-F}=23.7 \mathrm{~Hz}\right), 56.4,23.2,20.8$.

$\mathrm{UV}: \lambda_{\max }(\mathrm{MeOH})=206(\varepsilon=15606) \mathrm{nm}$.

\section{(R)-1-(1-Cyclohexylethyl)-3-((3-fluoro-4-piperidin-1-yl)phe-} nyl)thiourea (22a)

The isothiocyanate 11a $(0.08 \mathrm{~g}, 0.47 \mathrm{mmol})$ was dissolved in anhydrous $\mathrm{MeOH}(10 \mathrm{~mL})$ and an equimolar quantity of aromatic amine 21 $(0.09 \mathrm{~g}, 0.46 \mathrm{mmol})$ was added with stirring. The reaction mixture was heated to reflux for $4 \mathrm{~h}$. The reaction mixture was cooled and poured into ice cold water, where thiourea was precipitated. The solid product was washed with water and purified by column chromatography using petroleum ether and EtOAc (7:3) with $1 \%$ triethylamine as the eluent, which, on evaporation, afforded 22a.

Yield: $0.05 \mathrm{~g}$ (28\%); amorphous solid; $[\alpha]_{\mathrm{D}}+32.0$ (c 0.5, $\left.\mathrm{CHCl}_{3}\right)$.

IR (thin film): 3268 (NH), 2928, 2852, 1509 (Ar), 1450, 1335 (CS), $1232,1250(\mathrm{CF}), 1139,919,861,700 \mathrm{~cm}^{-1}$.

${ }^{1} \mathrm{H}$ NMR $\left(600 \mathrm{MHz}, \mathrm{CDCl}_{3}\right): \delta=7.6(\mathrm{~d}, J=8.2 \mathrm{~Hz}, \mathrm{NH}), 6.9(\mathrm{t}, J=9.0 \mathrm{~Hz}$, $1 \mathrm{H}), 6.9(\mathrm{~m}, 2 \mathrm{H}), 5.7(\mathrm{~s}, \mathrm{br}, \mathrm{NH}), 4.4(\mathrm{~m}, 1 \mathrm{H}), 3.0(\mathrm{~m}, 4 \mathrm{H}), 1.8-1.0(\mathrm{~m}$, $20 \mathrm{H})$.

${ }^{13} \mathrm{C}$ NMR $\left(150 \mathrm{MHz}, \mathrm{CDCl}_{3}\right): \delta=179.9,155.6\left(\mathrm{~J}_{\mathrm{C}-\mathrm{F}}=249.7 .0 \mathrm{~Hz}\right), 140.8$, 129.1, 121.9, 119.9, $114.0\left(J_{\mathrm{C}-\mathrm{F}}=22.4 \mathrm{~Hz}\right), 55.8,51.9(2 \mathrm{C}), 42.9,29.7$, 29.3, 28.9, 26.4, 26.0, 24.1, 17.3.

HRMS: $m / z$ calcd for $\mathrm{C}_{20} \mathrm{H}_{29} \mathrm{FN}_{3} \mathrm{~S}$ : 362.2061 ; found: 362.2047 .

(R)-N-(1-Cyclohexylethyl)-5-fluoro-6-(piperidin-1-yl)benzo[d]thiazol-2-amine (23a)

Thiourea 22a $(0.12 \mathrm{~g}, 0.33 \mathrm{mmol})$ was dissolved in chloroform ( $15 \mathrm{~mL})$ and the reaction mixture was cooled in an ice bath. Subsequently, an equimolar quantity of $\mathrm{Br}_{2}(0.017 \mathrm{~mL})$, in $\mathrm{CHCl}_{3}(3.4 \mathrm{~mL})$ was added to the reaction mixture. The ice bath was removed and the reaction mixture was brought to r.t. and stirred at r.t. for $18 \mathrm{~h}$. The resulting solution was neutralized with saturated $\mathrm{Na}_{2} \mathrm{CO}_{3}$ and extracted with $\mathrm{CH}_{2} \mathrm{Cl}_{2}(3 \times 10 \mathrm{~mL})$. The combined organic phases were dried over $\mathrm{Na}_{2} \mathrm{SO}_{4}$ and concentrated in vacuo. The resulting residue was purified by chromatography, eluting with petroleum ether and EtOAc (7:3) containing $1 \%$ triethylamine. The combined fractions were evaporated to afford 23a.

Yield: $30 \mathrm{mg}$ (29\%); amorphous solid; $[\alpha]_{\mathrm{D}}+35.5\left(\right.$ c 0.2, $\left.\mathrm{CHCl}_{3}\right)$.

IR (thin film): 3219 (NH), 2928, 2852, 1615 (Ar), 1578, 1471 (CN thiazole), 1450, 1382 (CN), 1338, 1275, 1227 (CF), 1203, 924, 855, $742 \mathrm{~cm}^{-1}$.

${ }^{1} \mathrm{H}$ NMR $\left(600 \mathrm{MHz}, \mathrm{CDCl}_{3}\right): \delta=7.2(\mathrm{~m}, 2 \mathrm{H}), 5.7$ (s, br, NH), 3.5 (app p, $J=5.7,5.7,5.6,5.6 \mathrm{~Hz}, 1 \mathrm{H}), 2.9(\mathrm{~m}, 4 \mathrm{H}), 1.8-1.0(\mathrm{~m}, 20 \mathrm{H})$.

${ }^{13} \mathrm{C}$ NMR $\left(150 \mathrm{MHz}, \mathrm{CDCl}_{3}\right): \delta=167.9,155.7\left(\mathrm{~J}_{\mathrm{C}-\mathrm{F}}=243.3 \mathrm{~Hz}\right), 146.6$, $136.7,124.6,111.0\left(J_{\mathrm{C}-\mathrm{F}}=3.6 \mathrm{~Hz}\right), 106.0\left(J_{\mathrm{C}-\mathrm{F}}=24.5 \mathrm{~Hz}\right), 57.3,53.0\left(\mathrm{~J}_{\mathrm{C}-\mathrm{F}}=\right.$ $2.5 \mathrm{~Hz}), 43.5,29.7,29.4,29.0,26.1,24.1,18.0$.

HRMS: $m / z$ calcd for $\mathrm{C}_{20} \mathrm{H}_{28} \mathrm{FN}_{3} \mathrm{NaS}$ : 384.1886; found: 384.1870 . 
(R)-1-(3-Fluoro-4-(piperidin-1-yl)phenyl)3-(1-phenylethyl)thiourea (22b)

Isothiocyanate $\mathbf{1 1 b}(0.08 \mathrm{~mL}, 0.49 \mathrm{mmol})$ was dissolved in anhydrous $\mathrm{MeOH}(10 \mathrm{~mL}$ ) and an equimolar quantity of aromatic amine $\mathbf{2 1}$ (90 $\mathrm{mg}, 0.46 \mathrm{mmol}$ ) was added with stirring. The reaction mixture was heated to reflux for $4 \mathrm{~h}$ at $65^{\circ} \mathrm{C}$, then concentrated in vacuo. The resultant mixture was redissolved in EtOAc $(20 \mathrm{~mL})$ and washed with water $(3 \times 20 \mathrm{~mL})$. The EtOAc layer was dried over sodium sulphate and concentrated in vacuo. The resulting mixture was subjected to chromatography using petroleum ether and $\operatorname{EtOAc}(7: 3)$ as the eluent. The eluents were then evaporated to afford $\mathbf{2 2 b}$.

Yield: $33 \mathrm{mg}$ (22\%); solid; $[\alpha]_{\mathrm{D}}-59.3\left(\mathrm{c} 0.7, \mathrm{CHCl}_{3}\right)$.

IR (thin film): 3253 (NH), 2934, 2853, 1509, 1451, 1332 (CS), 1250 (CF), 1232, 1136, 1121, 1025, 911, 861, 758, 725, $699 \mathrm{~cm}^{-1}$.

${ }^{1} \mathrm{H}$ NMR (600 MHz, $\mathrm{CDCl}_{3}$ ): $\delta=7.8(\mathrm{~s}, \mathrm{br}, \mathrm{NH}), 7.3(\mathrm{t}, J=7.5 \mathrm{~Hz}, 2 \mathrm{H}$ ), $7.2(\mathrm{~m}, 3 \mathrm{H}), 6.9(\mathrm{t}, J=8.7 \mathrm{~Hz}, 1 \mathrm{H}), 6.8(\mathrm{~m}, 2 \mathrm{H}), 6.1(\mathrm{~s}, \mathrm{NH}), 5.6(\mathrm{~m}$, $1 \mathrm{H}), 3.0(\mathrm{~m}, 4 \mathrm{H}), 1.7(\mathrm{~m}, 4 \mathrm{H}), 1.6(\mathrm{dt}, J=5.9,5.9,11.7 \mathrm{~Hz}, 2 \mathrm{H}), 1.5(\mathrm{~d}$, $J=6.9 \mathrm{~Hz}, 3 \mathrm{H})$.

${ }^{13} \mathrm{C}$ NMR $\left(150 \mathrm{MHz}, \mathrm{CDCl}_{3}\right): \delta=180.0,155.5\left(J_{\mathrm{C}-\mathrm{F}}=49.7 \mathrm{~Hz}\right), 142.2$, $140.7\left(J_{C-F}=9.3 \mathrm{~Hz}\right), 135.1,128.8,127.6,126.1,121.7\left(J_{C-F}=2.6 \mathrm{~Hz}\right)$, $119.8,113.9\left(J_{C-F}=22.8 \mathrm{~Hz}\right), 54.3,51.8\left(J_{C-F}=3.4 \mathrm{~Hz}\right), 26.0,24.1,17.6$.

UV: $\lambda_{\max }(\mathrm{MeOH})=253(\varepsilon=17954)$.

HRMS: $m / z$ calcd for $\mathrm{C}_{20} \mathrm{H}_{24} \mathrm{FN}_{3} \mathrm{NaS}$ : 380.1567 ; found: 380.1573 .

\section{(R)-5-Fluoro- $N$-(1-phenylethyl)-6-(piperidin-1-yl)benzo[d]thi- azol-2-amine (23b)}

Thiourea 22b (0.14 g, $0.39 \mathrm{mmol})$ was dissolved in chloroform (15 $\mathrm{mL}$ ) an the reaction mixture was cooled in an ice bath. Subsequently, an equimolar quantity of $\mathrm{Br}_{2}(0.014 \mathrm{~mL})$ in $\mathrm{CHCl}_{3}(2.8 \mathrm{~mL})$ was added to the reaction mixture. The ice bath was removed and the reaction mixture was brought to r.t. and stirred at r.t. for $18 \mathrm{~h}$. The mixture was neutralized with saturated $\mathrm{NaHCO}_{3}$ and extracted with $\mathrm{CH}_{2} \mathrm{Cl}_{2}(3 \times 10$ $\mathrm{mL}$ ). The combined organic phases were dried over $\mathrm{Na}_{2} \mathrm{CO}_{3}$ and concentrated in vacuo. The resulting residue was purified by chromatography using petroleum ether and EtOAc (7:3) containing 1\% trimethylamine as the eluent. Evaporation of the solvents in vacuo afforded 23b.

Yield: $0.05 \mathrm{~g}$ (36\%); viscous oil; $[\alpha]_{\mathrm{D}}+69.8\left(\mathrm{c} 0.8, \mathrm{CHCl}_{3}\right)$.

IR (thin film): 3209 (NH), 2935, 2853, 1709, 1613 (Ar), 1574, 1534, 1466 (CN thiazole), 1450, 1356 (CN thiazole), 1276, 1226 (CF), 1144, $919,855,741 \mathrm{~cm}^{-1}$.

${ }^{1} \mathrm{H}$ NMR (300 MHz, $\left.\mathrm{CDCl}_{3}\right): \delta=7.3(\mathrm{~m}, 3 \mathrm{H}), 7.3(\mathrm{~m}, 2 \mathrm{H}), 7.1(\mathrm{~d}, J=$ $12.9 \mathrm{~Hz}, 1 \mathrm{H}), 7.1(\mathrm{~d}, J=8.1 \mathrm{~Hz}, 1 \mathrm{H}), 6.4(\mathrm{~s}, \mathrm{br}, \mathrm{NH}), 4.7$ (q, $J=6.7,6.7$, $6.6 \mathrm{~Hz}, 1 \mathrm{H}), 2.9$ (m, $4 \mathrm{H}), 1.7$ (m, $4 \mathrm{H}), 1.6(\mathrm{~d}, J=6.8 \mathrm{~Hz}, 3 \mathrm{H}), 1.5(\mathrm{~m}$, $2 \mathrm{H})$.

${ }^{13} \mathrm{C}$ NMR $\left(150 \mathrm{MHz}, \mathrm{CDCl}_{3}\right): \delta=167.4,155.7\left(J_{\mathrm{C}-\mathrm{F}}=243.0 \mathrm{~Hz}\right), 146.7$ $\left(J_{\mathrm{C}-\mathrm{F}}=12.1 \mathrm{~Hz}\right), 142.8,136.9\left(J_{\mathrm{C}-\mathrm{F}}=11.3 \mathrm{~Hz}\right), 128.8,127.7,126.1,125.5$, $110.9\left(J_{C-F}=3.8 \mathrm{~Hz}\right), 106.3\left(J_{C-F}=24.6 \mathrm{~Hz}\right), 55.5,52.9,26.2,24.1,15.9$. HRMS: $m / z$ calcd for $\mathrm{C}_{20} \mathrm{H}_{23} \mathrm{FN}_{3} \mathrm{~S}$ : 356.1591; found: 356.1555 .

\section{1-(2-Fluoro-4-nitrophenyl)pyrrolidine (24)}

3,4-Difluoronitrobenzene 7 ( $2.86 \mathrm{~g}, 17.9 \mathrm{mmol})$ was dissolved in acetonitrile $(30 \mathrm{~mL})$, then pyrrolidine $(3.32 \mathrm{~g}, 46.54 \mathrm{mmol})$ was added and the mixture was heated at $82^{\circ} \mathrm{C}$ for $18 \mathrm{~h}$. The resultant solution was concentrated in vacuo. The residue was recrystallized from aqueous $\mathrm{MeOH}$ to give desired product 24 .

Yield: 3.22 g (86\%); yellow crystals; mp $134-137{ }^{\circ} \mathrm{C}$ [lit. ${ }^{32} \mathrm{mp} 137-$ $\left.138^{\circ} \mathrm{C}\right]$.
IR (thin film): 3500, 2980, 2884, 1607 (Ar), 1525, $1487\left(\mathrm{NO}_{2}\right), 1460$, 1481, $1300\left(\mathrm{NO}_{2}\right), 1285,1258$ (CF), 1207, 1160, 1077, 963, 880, 796, $742 \mathrm{~cm}^{-1}$.

${ }^{1} \mathrm{H}$ NMR $\left(400 \mathrm{MHz}, \mathrm{CDCl}_{3}\right): \delta=7.9(\mathrm{dd}, J=2.5,9.1 \mathrm{~Hz}, 1 \mathrm{H}), 7.8(\mathrm{dd}, J=$ 2.5, $14.2 \mathrm{~Hz}, 1 \mathrm{H}), 6.5(\mathrm{t}, J=8.9 \mathrm{~Hz}, 1 \mathrm{H}), 3.5(\mathrm{~m}, 4 \mathrm{H}), 2.0(\mathrm{~m}, 4 \mathrm{H})$.

${ }^{13} \mathrm{C} \mathrm{NMR}\left(75 \mathrm{MHz}, \mathrm{CDCl}_{3}\right): \delta=153.5\left(J_{\mathrm{C}-\mathrm{F}}=256.9 \mathrm{~Hz}\right), 147.3,138.4\left(J_{\mathrm{C}-\mathrm{F}}=\right.$ $5.4 \mathrm{~Hz}), 121.9\left(J_{\mathrm{C}-\mathrm{F}}=1.6 \mathrm{~Hz}\right), 112.9\left(J_{\mathrm{C}-\mathrm{F}}=1.9 \mathrm{~Hz}\right), 112.7\left(J_{\mathrm{C}-\mathrm{F}}=\right.$ $17.9 \mathrm{~Hz}), 50.0\left(\mathrm{~J}_{\mathrm{C}-\mathrm{F}}=5.8 \mathrm{~Hz}\right), 25.4$.

UV: $\lambda_{\max }(\mathrm{MeOH})=206(\varepsilon=22213) \mathrm{nm}$.

HRMS: $m / z$ calcd for $\mathrm{C}_{10} \mathrm{H}_{11} \mathrm{FN}_{2} \mathrm{NaO}_{2}$ : 233.0697; found: 233.0227.

\section{3-Fluoro-4-(pyrrolidin-1-yl)aniline (25) ${ }^{32}$}

Nitro compound 24 (0.60 g, $2.85 \mathrm{mmol})$ was dissolved in anhydrous $\mathrm{MeOH}(40 \mathrm{~mL})$ and the reaction flask was charged with $10 \%$ palladium on carbon and hydrogenated at $45-50$ psi for $72 \mathrm{~h}$. The reaction mixture was filtered through a plug of Celite and washed with $\mathrm{MeOH}$ $(100 \mathrm{~mL})$. Subsequent removal of the solvent in vacuo afforded 25.

Yield: $0.51 \mathrm{~g}$ (99\%); viscous oil.

IR (thin film): $3340(\mathrm{NH}), 3219(\mathrm{NH}), 2965,2873,1615$ (Ar), 1512, 1460, 1359, 1299, 1235 (CF), 1143, 950, 851, 799, $754 \mathrm{~cm}^{-1}$.

${ }^{1} \mathrm{H} \mathrm{NMR}\left(600 \mathrm{MHz}, \mathrm{CDCl}_{3}\right): \delta=7.3(\mathrm{~s}, \mathrm{br}, 2 \mathrm{H}, \mathrm{NH}), 6.6(\mathrm{dd}, J=8.5$, $9.7 \mathrm{~Hz}, 1 \mathrm{H}$ ), 6.4 (dd, $J=2.6,14.3 \mathrm{~Hz}, 1 \mathrm{H}), 6.4$ (ddd, $J=0.8,2.6,8.5 \mathrm{~Hz}$, $1 \mathrm{H}), 3.2(\mathrm{~m}, 4 \mathrm{H}), 1.9(\mathrm{~m}, 4 \mathrm{H})$.

${ }^{13} \mathrm{C}$ NMR $\left(150 \mathrm{MHz}, \mathrm{CDCl}_{3}\right): \delta=153.9\left(J_{\mathrm{C}-\mathrm{F}}=242.6 \mathrm{~Hz}\right), 139.1\left(J_{\mathrm{C}-\mathrm{F}}=\right.$ $10.0 \mathrm{~Hz}), 130.5\left(\mathrm{~J}_{\mathrm{C}-\mathrm{F}}=10.4 \mathrm{~Hz}\right), 117.0\left(J_{\mathrm{C}-\mathrm{F}}=5.8 \mathrm{~Hz}\right), 111.0,104.5\left(\mathrm{~J}_{\mathrm{C}-\mathrm{F}}=\right.$ $24.0 \mathrm{~Hz}), 50.4,45.4,29.7,24.6$.

\section{(R)-1-(1-Cyclohexylethyl)-3-(3-fluoro-4-(pyrrolidin-1-yl)phe- nyl)thiourea (22c)}

Isothiocyanate 11a $(0.05 \mathrm{~g}, 0.29 \mathrm{mmol})$ was dissolved in anhydrous $\mathrm{MeOH}(10 \mathrm{~mL})$ and an equimolar quantity of $25(0.05 \mathrm{~g}, 0.27 \mathrm{mmol})$ was then added with stirring under $\mathrm{N}_{2}$ atmosphere. The reaction mixture was heated to reflux for $3 \mathrm{~h}$ at $65{ }^{\circ} \mathrm{C}$, then cooled and poured into ice cold water, in order to precipitate the thiourea. The solid product was washed with water and purified by column chromatography using petroleum ether and EtOAc (7:3) as the eluent, which, on evaporation, gave the desired product $\mathbf{2 2 c}$.

Yield: $10 \mathrm{mg}$ (12\%); brown amorphous solid; $[\alpha]_{\mathrm{D}}+94.1\left(\mathrm{c} 0.3, \mathrm{CHCl}_{3}\right)$. IR (thin film): $3256(\mathrm{NH}), 2925,2850,1711,1620$ (Ar), 1510, 1487, 1363, 1298 (CS), 1248 (CF), 1139, 962, 865, $715 \mathrm{~cm}^{-1}$.

${ }^{1} \mathrm{H}$ NMR $\left(600 \mathrm{MHz}, \mathrm{CDCl}_{3}\right): \delta=7.3(\mathrm{~s}, \mathrm{br}, \mathrm{NH}), 6.8(\mathrm{~m}, 2 \mathrm{H}), 6.6(\mathrm{~m}$, $1 \mathrm{H}), 5.7$ (s, br, NH), $4.4(\mathrm{~m}, 1 \mathrm{H}), 3.4(\mathrm{~m}, 4 \mathrm{H}), 1.9(\mathrm{~m}, 4 \mathrm{H}), 1.8-0.9(\mathrm{~m}$, $14 \mathrm{H})$.

${ }^{13} \mathrm{C} \mathrm{NMR}\left(150 \mathrm{MHz}, \mathrm{CDCl}_{3}\right): \delta=180.4,151.5\left(J_{\mathrm{C}-\mathrm{F}}=244.9 \mathrm{~Hz}\right), 137.1\left(J_{\mathrm{C}-\mathrm{F}}=\right.$ $9.4 \mathrm{~Hz}), 122.9\left(J_{\mathrm{C}-\mathrm{F}}=2.3 \mathrm{~Hz}\right), 115.4\left(J_{\mathrm{C}-\mathrm{F}}=6.5 \mathrm{~Hz}\right), 114.8,102.4,55.7$, $49.7\left(J_{C-F}=5.1 \mathrm{~Hz}\right), 42.9,29.3,28.9,26.4,26.1,26.0,25.3,17.3$.

HRMS: $m / z$ calcd For $\mathrm{C}_{19} \mathrm{H}_{28} \mathrm{FN}_{3} \mathrm{NaS}$ : 372.1880 ; found: 372.1850 .

\section{(R)-N-(1-Cyclohexylethyl)-5-fluoro-6-(pyrrolidin-1-yl)ben- zo[d]thiazol-2-amine (23c)}

Thiourea 22c $(0.07 \mathrm{~g}, 0.20 \mathrm{mmol})$ was dissolved in chloroform (15 mL) and the reaction mixture was cooled in an ice bath. Subsequently, an equimolar quantity of $\mathrm{Br}_{2}(0.01 \mathrm{~mL})$ in $\mathrm{CHCl}_{3}(2 \mathrm{~mL})$ was added to the reaction mixture. The ice bath was removed and the reaction mixture was brought to r.t. and stirred at r.t. for $18 \mathrm{~h}$. The resulting solution was neutralized with saturated $\mathrm{NaHCO}_{3}$ and extracted with $\mathrm{CH}_{2} \mathrm{Cl}_{2}$ $(3 \times 10 \mathrm{~mL})$. The combined organic phases were dried over $\mathrm{Na}_{2} \mathrm{SO}_{4}$ 
and concentrated in vacuo. The resulting residue was purified by chromatography with petroleum ether and $\operatorname{EtOAc}(7: 3)$ as the eluents. Removal of the solvents in vacuo gave the desired product $\mathbf{2 3 c}$.

Yield: $0.06 \mathrm{~g}$ (86\%); solid; $[\alpha]_{\mathrm{D}}+28.1\left(\right.$ c $\left.0.3, \mathrm{CHCl}_{3}\right)$.

IR (thin film): 3216 (NH), 2924, 2852, 1702, 1615 (Ar), 1578, 1539, 1470 (CN thiazole), 1358 (CN thiazole), 1290, 1249 (CF), 1131, 850, $748,700,677 \mathrm{~cm}^{-1}$.

${ }^{1} \mathrm{H} \mathrm{NMR}\left(300 \mathrm{MHz}, \mathrm{CDCl}_{3}\right): \delta=7.2(\mathrm{~d}, J=14.0 \mathrm{~Hz}, 1 \mathrm{H}), 6.9(\mathrm{~d}, J=$ $8.2 \mathrm{~Hz}, 1 \mathrm{H}), 5.1$ (s, br, NH), $3.5(\mathrm{~m}, 1 \mathrm{H}), 3.3(\mathrm{~m}, 4 \mathrm{H}), 1.9(\mathrm{~m}, 4 \mathrm{H}), 1.9-$ $0.8(\mathrm{~m}, 14 \mathrm{H})$.

${ }^{13} \mathrm{C}$ NMR $\left(150 \mathrm{MHz}, \mathrm{CDCl}_{3}\right): \delta=166.6,152.9\left(\mathrm{~J}_{\mathrm{C}-\mathrm{F}}=240.0 \mathrm{~Hz}\right), 144.1$, 133.6, 125.5, 106.6, 106.1, 56.9, 50.4, 43.6, 29.0, 26.4, 26.1, 24.9, 18.1. HRMS: $m / z$ calcd for $\mathrm{C}_{19} \mathrm{H}_{26} \mathrm{FN}_{3} \mathrm{NaS}$ : 370.1729; found: 370.1734 .

(R)-1-(3-Fluoro-4-(pyrrolidin-1-yl)phenyl)-3-(1-phenylethyl)thiourea (22d)

Isothiocyanate 11b $(0.21 \mathrm{~g}, 1.29 \mathrm{mmol})$ was dissolved in anhydrous $\mathrm{MeOH}(10 \mathrm{~mL})$ and an equimolar quantity of $25(0.23 \mathrm{~g}, 1.29 \mathrm{mmol})$ was added with stirring. The reaction mixture was heated for $3 \mathrm{~h}$ at $65^{\circ} \mathrm{C}$, then cooled and poured into ice-cold water, resulting in the thiourea being precipitated. The solid product was washed with water and purified by chromatography with petroleum ether and EtOAc $(7: 3)$ as the eluents. Subsequent evaporation of the solvents afforded 22d.

Yield: $0.26 \mathrm{~g}$ (59\%); amorphous solid; $[\alpha]_{\mathrm{D}}+138.6$ ( c 1.9, $\mathrm{CHCl}_{3}$ ).

IR (thin film): 3237 (NH), 2969, 2872, 1619 (Ar), 1514, 1486, 1364, 1303 (CS), 1232 (CF), 1179, 1147, 1022, 959, 864, 795, 757, $698 \mathrm{~cm}^{-1}$.

${ }^{1} \mathrm{H} \mathrm{NMR}\left(600 \mathrm{MHz}, \mathrm{CDCl}_{3}\right): \delta=7.7(\mathrm{~s}, \mathrm{NH}), 7.3(\mathrm{t}, J=7.5 \mathrm{~Hz}, 2 \mathrm{H}), 7.2$ $(\mathrm{m}, 3 \mathrm{H}), 6.8(\mathrm{~m}, 2 \mathrm{H}), 6.6(\mathrm{t}, J=9.3 \mathrm{~Hz}, 1 \mathrm{H}), 6.1(\mathrm{br}, \mathrm{NH}), 5.7(\mathrm{~m}, 1 \mathrm{H})$, $3.4(\mathrm{~m}, 4 \mathrm{H}), 1.9(\mathrm{~m}, 4 \mathrm{H}), 1.5(\mathrm{~d}, J=6.9 \mathrm{~Hz}, 3 \mathrm{H})$.

${ }^{13} \mathrm{C}$ NMR $\left(150 \mathrm{MHz}, \mathrm{CDCl}_{3}\right): \delta=180.4,151.3\left(\mathrm{~J}_{\mathrm{C}-\mathrm{F}}=244.7 \mathrm{~Hz}\right), 142.5$, $137.0\left(J_{C-F}=9.7 \mathrm{~Hz}\right), 128.7,127.4,126.1,125.7,122.7,115.4\left(J_{C-F}=\right.$ $6.0 \mathrm{~Hz}), 114.6,54.1,49.7\left(J_{\mathrm{C}-\mathrm{F}}=5.1 \mathrm{~Hz}\right), 25.3\left(\mathrm{~J}_{\mathrm{C}-\mathrm{F}}=1.7 \mathrm{~Hz}\right), 21.6$.

HRMS: $m / z$ calcd for $\mathrm{C}_{19} \mathrm{H}_{21} \mathrm{FN}_{3} \mathrm{~S}$ : 342.1435; found: 342.1422 .

\section{(R)-5-Fluoro- $N$-(1-phenylethyl)-6-(pyrrolidin-1-yl)benzo[d]thi-} azol-2-amine (23d)

Thiourea 22d ( $0.16 \mathrm{~g}, 0.48 \mathrm{mmol})$ was dissolved in chloroform (15 $\mathrm{mL}$ ) and the reaction mixture was cooled in an ice bath. Subsequently, an equimolar quantity of $\mathrm{Br}_{2}(0.03 \mathrm{~mL})$ in $\mathrm{CHCl}_{3}(6 \mathrm{~mL})$ was added to the reaction mixture. The ice bath was removed and the reaction mixture was brought to r.t. and stirred at r.t. for $18 \mathrm{~h}$. The resulting solution was neutralized with saturated $\mathrm{NaHCO}_{3}$ and extracted with $\mathrm{CH}_{2} \mathrm{Cl}_{2}(3 \times 10 \mathrm{~mL})$. The combined organic phases were dried over $\mathrm{Na}_{2} \mathrm{SO}_{4}$ and concentrated in vacuo. The resulting residue was subjected to column chromatography, eluting with toluene and EtOAc (9:1). Removal of the solvents in vacuo gave the desired product 23d.

Yield: $0.03 \mathrm{~g}(21 \%) ;[\alpha]_{\mathrm{D}}+16.9\left(\mathrm{c} 0.7, \mathrm{CHCl}_{3}\right)$.

IR (thin film): 3208 (NH), 2969, 2873, 1615 (Ar), 1536, 1469 (CN thiazole), 1356 (CN thiazole), 1249 (CF), 1128, 960, 851, 761, $699 \mathrm{~cm}^{-1}$.

${ }^{1} \mathrm{H} \mathrm{NMR}\left(600 \mathrm{MHz}, \mathrm{CDCl}_{3}\right): \delta=7.4(\mathrm{~m}, 2 \mathrm{H}), 7.3(\mathrm{~m}, 2 \mathrm{H}), 7.2(\mathrm{~m}, 1 \mathrm{H})$, $7.1(\mathrm{~d}, J=13.9 \mathrm{~Hz}, 1 \mathrm{H}), 6.8(\mathrm{~d}, J=8.3 \mathrm{~Hz}, 1 \mathrm{H}), 5.9(\mathrm{~s}, \mathrm{br}, \mathrm{NH}), 4.7(\mathrm{q}, J=$ 6.7, 6.7, $6.7 \mathrm{~Hz}, 1 \mathrm{H}), 3.3(\mathrm{~s}, 4 \mathrm{H}), 1.9(\mathrm{~m}, 4 \mathrm{H}), 1.6(\mathrm{~d}, J=6.7 \mathrm{~Hz}, 3 \mathrm{H})$.

${ }^{13} \mathrm{C}$ NMR $\left(150 \mathrm{MHz}, \mathrm{CDCl}_{3}\right): \delta=165.8,152.8\left(\mathrm{~J}_{\mathrm{C}-\mathrm{F}}=241.2 \mathrm{~Hz}\right), 142.9$, 134.7, 128.8, 127.6, 127.5, 126.1, 106.6 (2C), $106.5\left(J_{C-F}=5.4 \mathrm{~Hz}\right), 55.3$, $50.4\left(J_{\mathrm{C}-\mathrm{F}}=4.7 \mathrm{~Hz}\right), 24.9\left(J_{\mathrm{C}-\mathrm{F}}=1.3 \mathrm{~Hz}\right), 23.7$.

HRMS: $m / z$ calcd for $\mathrm{C}_{19} \mathrm{H}_{19} \mathrm{FN}_{3} \mathrm{~S}$ : 340.1284 ; found: 340.1293 .

\section{Funding Information}

We thank to the government of Trinidad and Tobago and University of the West Indies for financial support.

\section{Acknowledgment}

We thank Joseph Maddry, NIH, for the biological assessments and Prof. C. Abell for insightful discussions.

\section{Supporting Information}

Supporting information for this article is available online at https://doi.org/10.1055/s-0040-1707105.

\section{References and Notes}

(1) World Health Organization. November 2019. Tuberculosis: WHO factsheet no 104. Available: https://www.who.int/tb/publications/factsheets/en/ [Accessed 26.02.2020]

(2) (a) Daniel, T. M.; Bates, J. H.; Downes, K. A. In History of Tuberculosis. Tuberculosis: Pathogenesis, Protection and Control; Bloom, B., Ed.; ASM Press: Washington DC, 1994, 13-24. (b) Sensi, P.; Grassi, G. G. In Burger's Medicinal Chemistry and Drug Discovery; Abraham, D. J., Ed.; John Wiley \& Sons Inc: Weinheim, 2003, 821-824. (c) Janin, Y. L. Bioorg. Med. Chem. 2007, 15, 2479. (d) Ballell, L.; Field, R. A.; Duncan, K.; Young, R. J. Antimicrob. Agents Chemother. 2005, 49, 2153.

(3) (a) Snider, D. E. J.; Castro, K. G. N. Engl. J. Med. 1998, 338, 1689. (b) Chan, E. D.; Iseman, M. D. Br. Med. J. 2002, 325, 1282. (c) Bastian, I.; Colebunders, R. Drugs 1999, 58, 633. (d) Basso, L. A.; Blanchard, J. S. Adv. Exp. Med. Biol. 1998, 456, 115. (e) Duncan, K.; Barry, C. E. III. Curr. Opin. Microbiol. 2004, 7, 460. (f) Blanchard, J. S. Annu. Rev. Biochem. 1996, 65, 215. (g) Iseman, M. D. N. Engl. J. Med. 1993, 329, 784. (h) Farmer, P.; Kim, J. Y. Br. Med. J. 1998, 317, 671.

(4) Rana, A.; Siddiqui, N.; Khan, S. A. Indian J. Pharm. Sci. 2007, 69, 10.

(5) Suter, H.; Zutter, H. Helv. Chim. Acta 1967, 50, 1084.

(6) (a) He, Y.; Benz, A.; Fu, T.; Wang, M.; Covey, D. F.; Zorumski, C. F.; Mennick, S. Neuropharmacology 2002, 42, 199. (b) Jimonet, P.; Audiau, F.; Barreau, M.; Blanchard, J.-C.; Boireau, A.; Bour, Y.; Coleno, M.-A.; Doble, A.; Doerflinger, G.; Huu, C. D.; Donat, M.H.; Duchesne, J. M.; Ganil, P.; Gueremy, C.; Honore, E.; Just, B.; Kerphirique, R.; Gontier, S.; Hubert, P.; Laduron, P. M.; Le Blevec, J.; Meunier, M.; Miquet, J.-M.; Nemecek, C.; Pasquet, M.; Piot, O.; Pratt, J.; Rataud, J.; Reibaud, M.; Stutzmann, J.-M.; Mignani, S. J. Med. Chem. 1999, 42, 2828. (c) Hays, S. J.; Rice, M. J.; Ortwine, D. F.; Johnson, G.; Schwartz, R. D.; Boyd, D. K.; Copeland, L. F.; Vartanian, M. G.; Boxer, P. A. J. Pharm. Sci. 1994, $83,1425$.

(7) (a) Foscolos, G.; Tsatsas, G.; Champagnac, A.; Pommier, M. Ann. Pharm. Fr. 1977, 35, 295. (b) Paris, J.; Conquelet, J.; Tronche, P.; Bastide, J.; Bastide, P. Chim. Ther. 1973, 8, 655. (c) Yukichi, K.; Yuji, I.; Koichi, H. Japanese Patent JP 50018463, 1976; Chem. Abstr. 1975, 83, 58798.

(8) (a) Khedekar, P. B.; Bahekar, R. H.; Chopade, R. S.; Umathe, S. N.; Rao, A. R. R.; Bhusari, K. P. Arzneim. Forsch. 2003, 53, 640.

(9) (a) Mishra, A. L. J. Org. Chem. 1958, 23, 1388. 
(10) (a) Sawhney, S. N.; Arora, S. K.; Singh, J. V.; Bansal, O. P.; Singh, S. P. Indian J. Chem., Sect. B: Org. Chem. Incl. Med. Chem. 1978, 16, 605. (b) Paget, C. J.; Kisner, K.; Stone, R. L.; Delong, D. C. J. Med. Chem. 1969, 12, 1016.

(11) (a) Korpe, G. V.; Deshmukh, S. P.; Fokmare, A. K. Indian J. Heterocycl. Chem. 2001, 10, 287. (b) Lacova, M.; Chovancova, J.; Hyblova, O.; Varkonda, S. Chem. Pap. 1991, 45, 411. (c) Capek, A.; Svab, A.; Budesinsky, Z. Folia Microbiologica 1976, 21, 152.

(12) (a) Mehra, S. C.; Zaman, S.; Khan, A. A. J. Indian Chem. Soc. 1980, 57, 829. (b) Srivastava, P. K.; Rai, S. K. Quarterly J. Surgical Sci. 1979, 15, 73.

(13) Caleta, I.; Grdisa, M.; Mrvos-Sermek, D.; Cetina, M.; Tralic-Kulenovic, V.; Pavelic, K.; Karmainski-Zamola, G. Farmaco 2004, 59, 297.

(14) (a) Rathod, A. S.; Berad, B. N.; Doshi, A. G. Orient. J. Chem. 2000, 16, 549. (b) El-Shaaer, H. M.; Foltinova, P.; Lacova, M.; Chovancova, J.; Stankovicova, H. Farmaco 1998, 53, 224.

(15) Yoshida, M.; Hayakawa, I.; Hayashi, N.; Agatsuma, T.; Oda, Y.; Tansawa, F.; Iwasaki, S.; Koyama, K.; Furukawa, H.; Kurakata, S.; Sugano, Y. Bioorg. Med. Chem. Lett. 2005, 15, 3328.

(16) (a) Bhusari, K. P.; Khedekar, P. B.; Umathe, S. N.; Bahekar, R. H.; Rao, A. R. R. Indian J. Heterocycl. Chem. 2000, 9, 213. (b) Shirke, V. G.; Bobade, A. S.; Bhamaria, R. P.; Khadse, B. G.; Sengupta, S. R. Indian Drugs 1990, 27, 350. (c) Gvozdjakova, A.; Petrakova, E.; Odlerova, Z. Czech Patent CS182708, 1978; Chem. Abstr. 1981, 94, 156914.

(17) (a) Mahajan, A.; Yeh, S.; Nell, M.; van Rensburg, C. E. J.; Chibale, K. Bioorg. Med. Chem. Lett. 2007, 17, 5683. (b) Venkatachalam, T. K.; Vassilev, A. O.; Benyunov, A.; Grigoriants, O. O.; Tibbles, H. E.; Uckun, F. M. Lett. Drug Des. Discovery 2007, 4, 318. (c) AbdelRahman, H. M.; Morsy, M. A. J. Enzyme Inhib. Med. Chem. 2007, 22, 57. (d) Li, J.; Tan, J.-z.; Chen, L.-l.; Zhang, J.; Shen, X.; Mei, C.l.; Fu, L.-l.; Lin, L.-p.; Ding, J.; Xiong, B.; Xiong, X.-S.; Liu, H.; Luo, X.-m.; Jiang, H.-l. Acta Pharmacol. Sinica 2006, 27, 1259. (e) Figueiredo, I. M.; dos Santos, L. V.; da Costa, W. F.; de Carvalho, J. E.; da Silva, C. C.; Sacoman, J. L.; Kohn, L. K.; Sarragiotto, M. H. J. Braz. Chem. Soc. 2006, 17, 954. (f) Esteves-Souza, A.; Pissinate, K.; Nascimento Mda, G.; Grynberg, N. F.; Echevarria, A. Bioorg. Med. Chem. 2006, 14, 492.

(18) Matteelli, A.; Carvalho, A. C.; Dooley, K. E.; Kritski, A. Future Microbiol. 2010, 5, 849.
(19) Haagsma, A. C.; Abdillahi-Ibrahim, R.; Wagner, M. J.; Krab, K.; Vergauwen, K.; Guillemont, J.; Andries, K.; Lill, H.; Koul, A.; Bald, D. Antimicrob. Agents Chemother. 2009, 53, 1290.

(20) Manjula, S. N.; Noolvi, M. N.; Parihar, V. K.; Reddy, M. S. A.; Raman, V.; Gadad, A. K.; Singh, G.; Kutty, N. G.; Rao, M. C. Eur. J. Med. Chem. 2009, 44, 2923.

(21) (a) Sriram, D.; Yogeeswari, P.; Madhu, K. Bioorg. Med. Chem. Lett. 2006, 16, 876. (b) Dixit, P. P.; Patil, V. J.; Nair, P. S.; Jain, S.; Sinha, N.; Arora, S. K. Eur. J. Med. Chem. 2006, 41, 423. (c) Karaku, S.; Rollas, S. Farmaco 2002, 577. (d) Küçükgüzel, I.; Küçükgüzel, S. G.; Rollas, S.; Kiraz, M. Bioorg. Med. Chem. Lett. 2001, 11, 1703.

(22) Maienfisch, P.; Hall, R. G. CHIMIA Int. J. Chem. 2004, 58, 93.

(23) Edmonds, M.; Peddie, V. Chem. N. Z. 2006, 85.

(24) (a) Patočka, J.; Dvořák, A. J. Appl. Biomed. 2004, 2, 95. (b) Caner, H.; Groner, E.; Levy, L.; Agranat, I. Drug Discovery Today 2004, 9, 105. (c) Waldeck, W. Chirality 2004, 5, 350. (d) Schaus, J. V. Chem. Eng. News 2000, 78, 55.

(25) (a) Talath, S.; Gadad, A. K. Eur. J. Med. Chem. 2006, 41, 918. (b) Gadad, A. K.; Noolvi, M. N.; Karpoormath, R. V. Bioorg. Med. Chem. 2004, 12, 5651. (c) Gadad, A. K.; Karki, S. S.; Rajurkar, V. G.; Bhongade, B. A. Arzneim. Forsch. 1999, 49, 858.

(26) Sharma, S. Synthesis 1978, 803.

(27) Jordan, A. D.; Luo, C.; Reitz, A. B. J. Org. Chem. 2003, 68, 8693.

(28) (a) Bestgen, B.; Krimm, I.; Kufareva, I.; Kamal, A. A. M.; Seetoh, W.-G.; Abell, C.; Hartmann, R. W.; Abagyan, R.; Cochet, C.; Le Borgne, M.; Engel, M.; Lomberget, T. J. Med. Chem. 2019, 62, 1803. (b) Bestgen, B.; Kufareva, I.; Seetoh, W.-G.; Abell, C.; Hartmann, R. W.; Abagyan, R.; Le Borgne, M.; Filhol, O.; Cochet, C.; Lomberget, T.; Engel, M. J. Med. Chem. 2019, 62, 1817.

(29) Brickner, S. J.; Hutchinson, D. K.; Barbachyn, M. R.; Manninen, P. R.; Ulanowicz, D. A.; Garmon, S. A.; Grega, K. C.; Hendges, S. K.; Toops, D. S.; Ford, C. W.; Zurenko, G. E. J. Med. Chem. 1996, 39, 673.

(30) (a) Tsogoeva, S. B.; Yalalov, D. A.; Hateley, M. J.; Weckbecker, C.; Huthmacher, K. Eur. J. Org. Chem. 2005, 4995. (b) Kjaer, A. Acta Chem. Scand. 1957, 11, 184. (c) Tsogoeva, S. B.; Hateley, M. J.; Yalalov, D. A.; Meindl, K.; Weckbecker, C.; Huthmacher, K. Bioorg. Med. Chem. 2005, 13, 5680.

(31) Janakiramudu, D. B.; Rao, D. S.; Srikanth, C.; Madhusudhana, S.; Murthy, P. S.; Nagalakshmidevamma, M.; Chalapathi, P. V.; Raju, C. N. Res. Chem. Intermed. 2018, 44, 469.

(32) (Sunshine Lake Pharma Co. Ltd.) AR Patent 92240, 2015. 